\title{
Clustering coefficient of random intersection graphs with infinite degree variance
}

\author{
Mindaugas Bloznelis and Valentas Kurauskas
}

Vilnius university, Lithuania

Dedicated to Professor Michat Karonski on the occasion of his 70th birthday

\begin{abstract}
For a random intersection graph with a power law degree sequence having a finite mean and an infinite variance we show that the global clustering coefficient admits a tunable asymptotic distribution.
\end{abstract}

key words: clustering coefficient, power law, infinite variance, random intersection graph, affiliation network.

\section{Introduction}

The global clustering coefficient $C_{G}$ of a graph $G$ is the ratio $C_{G}=3 \Delta / \Lambda$, where $\Delta$ is the number of triangles and $\Lambda$ is the number of paths of length 2. Another way to represent the global clustering coefficient is by the conditional probability that a randomly chosen triple of vertices makes up a triangle given that the first two vertices are adjacent to the third one. Formally,

$$
C_{G}=\mathbf{P}^{*}\left(v_{1}^{*} \sim v_{2}^{*} \mid v_{1}^{*} \sim v_{3}^{*}, v_{2}^{*} \sim v_{3}^{*}\right),
$$

where $\left(v_{1}^{*}, v_{2}^{*}, v_{3}^{*}\right)$ is an ordered triple of vertices sampled uniformly at random and the probability $\mathbf{P}^{*}$ refers to the sampling. By $\sim$ we denote the adjacency relation.

In this paper we study the relation between the clustering coefficient and the tail of the degree sequence in large complex networks. We focus on random intersection graph models of real affiliation networks (mode two networks), [12, , 9], 3]. They admit tunable degree distribution and non-vanishing clustering coefficient [14, [6], 1], 4]. Definition of a random intersection graph is recalled below in this section.

The global clustering coefficient $C_{G}$ of a realised instance $G$ of a random graph is a random variable. We note that generally this random variable behaves differently depending on whether the degree variance is finite or infinite [1], [11, [17. When the degree variance is finite the global clustering coefficient $C_{G}$ can be approximated by the corresponding numerical characteristic of the underlying random intersection graph model, the conditional probability $\alpha_{C}:=\mathbf{P}\left(v_{1}^{*} \sim\right.$ $\left.v_{2}^{*} \mid v_{1}^{*} \sim v_{3}^{*}, v_{2}^{*} \sim v_{3}^{*}\right)$, [13]. Here and below $\mathbf{P}$ refers to all the sources of randomness defining the events considered (these are the uniform sampling of vertices $\left(v_{1}^{*}, v_{2}^{*}, v_{3}^{*}\right)$ and random graph generation mechanism in the present context). We remark that $\alpha_{C}$ admits a simple asymptotic expression in terms of the first and second moment of the degree sequence [1, [10, [4], [5]. The question about the behaviour of the clustering coefficient $C_{G}$ when the degree variance is infinite remained open. We address this question in the present paper. Our study is analytical. 
For an infinite degree variance we show that $C_{G}$ admits a non-degenerate asymptotic distribution with tunable characteristics in the case where the weights defining the underlying random intersection graph achieve a certain balance. In this way our theoretical findings contribute to the discussion about whether and when a power law network model with an infinite degree variance can have a non-vanishing global clustering coefficient, cf. [17], where a negative result was obtained.

The paper is organized as follows. In this section we introduce random intersection graphs, formulate and discuss our results. Proofs are given in section 2. Technical lemmas are postponed to Section 3.

\subsection{Random intersection graphs}

Random intersection graphs model social networks, where the actors establish communication links provided that they share some common attributes (collaboration networks, actor networks, etc.). A random intersection graph $G$ on the vertex set $V=\left\{v_{1}, \ldots, v_{n}\right\}$ is defined by a random bipartite graph, denoted by $H$, with the bipartition $V \cup W$, where $W=\left\{w_{1}, \ldots, w_{m}\right\}$ is an auxiliary set of attributes. Two vertices in $G$ are adjacent whenever they have a common neighbour in $H$. This neighbour is called a witness of the adjacency relation.

In the active graph, denoted by $G(n, m, \mathrm{P})$, vertices $v \in V$ select their neighbourhoods $S_{v} \subset$ $W$ in $H$ independently at random according to the probability distribution $\mathbf{P}\left(S_{v}=A\right)=$ $\mathrm{P}(|A|)\left(\begin{array}{c}m \\ |A|\end{array}\right)^{-1}, A \subset W$. Here $\mathrm{P}$ is the probability distribution modeling the size $\left|S_{v}\right|$ of the neighbourhood of $v$ in $H$. Given the size $\left|S_{v}\right|$, the elements of $S_{v}$ are selected uniformly at random. Two vertices $u, v$ are adjacent in $G$ whenever the random sets $S_{u}$ and $S_{v}$ (called attribute sets of $u$ and $v$ ) intersect.

In the passive graph, denoted by $G^{\star}\left(n, m, \mathrm{P}^{\star}\right)$, attributes $w \in W$ select their neighbourhoods $D_{w} \subset V$ in $H$ independently at random according to the probability distribution $\mathbf{P}\left(D_{w}=A\right)=$ $\mathrm{P}^{\star}(|A|)\left(\begin{array}{c}n \\ |A|\end{array}\right)^{-1}, A \subset V$. Two vertices $u, v$ are adjacent in $G^{\star}\left(n, m, \mathrm{P}^{\star}\right)$ whenever $u, v \in D_{w}$ for some $w \in W$.

The inhomogeneous graph, denoted by $G\left(n, m, \mathrm{P}_{X}, \mathrm{P}_{Y}\right)$, interpolates between the active and passive models. It is defined by the random bipartite graph, where attributes $w_{i} \in W$ and vertices $v_{j} \in V$ are assigned independent random weights $X_{i}$ and $Y_{j}$ respectively. The weights model the attractiveness of attributes and activity of actors. Every pair $\left(w_{i}, v_{j}\right) \in W \times V$ is linked in $H$ with probability $p_{i j}=\min \left\{1, X_{i} Y_{j} / \sqrt{m n}\right\}$ independently of the other pairs. Here $X_{1}, \ldots, X_{m}$ and $Y_{1}, \ldots, Y_{n}$ are non-negative independent random variables with the distributions $\mathrm{P}_{X}$ and $\mathrm{P}_{Y}$ respectively.

In what follows we assume that $n / m$ is bounded and it is bounded away from zero as $m, n \rightarrow+\infty$, denoted by $n=\Theta(m)$. The rationale behind this assumption is that in the range $n=\Theta(m)$ the active, passive and inhomogeneous models admit non-degenerate asymptotic degree distributions including power laws [1, 2, 4, 6]. More importantly, in this range these random graph models admit tunable global clustering coefficient $C_{G} \approx \alpha_{C}$, provided that the degree variance is finite [13. Therefore it is reasonable to consider the range $n=\Theta(m)$, also when studying the global clustering coefficient of a power law intersection graph with an infinite degree variance.

\subsection{Results}

Let $d\left(v_{i}\right)$ denote the degree of a vertex $v_{i} \in V=\left\{v_{1}, \ldots, v_{n}\right\}$ in a random intersection graph. We note that the random variables $d\left(v_{1}\right), \ldots, d\left(v_{n}\right)$ are identically distributed for each particular model: active, passive and inhomogeneous. When speaking about the asymptotic degree 
distribution below we think about the limit in distribution of the random variable $d\left(v_{1}\right)$ as $n, m \rightarrow+\infty$.

Active graph $G=G(n, m, P)$. In Theorem 1 below we show that an active graph with an infinite degree variance has the global clustering coefficient $C_{G} \approx 0$.

Theorem 1. Let $\beta>0$. Let $m, n \rightarrow+\infty$. Assume that $m / n \rightarrow \beta$. Let $Z$ be a non-negative random variable such that $\mathbf{E} Z<\infty$ and $\mathbf{E} Z^{2}=\infty$. Let $P$ denote the distribution of $\min \{Z, m\}$. The global clustering coefficient of the active random graph $G(n, m, P)$ satisfies $C_{G}=o_{P}(1)$.

Under conditions of Theorem 1 the active graph has a mixed Poisson asymptotic degree distribution assigning probabilities $\mathbf{E} e^{-\lambda} \frac{\lambda^{k}}{k !}$ to the integers $k=0,1, \ldots$, see [1]. Here $\lambda=(\mathbf{E} Z) \beta^{-1} Z$ is a random variable. In the case where $Z$ has a power law with the tail index $\alpha>1$, i.e., for some $c_{z}>0$ we have

$$
\mathbf{P}(Z>t)=c_{z} t^{-\alpha}+o\left(t^{-\alpha}\right) \quad \text { as } \quad t \rightarrow+\infty,
$$

the asymptotic degree distribution described above is a power law with the same tail index $\alpha$. For $1<\alpha \leq 2$ it has a finite first moment, infinite variance and the clustering coefficient $C_{G} \approx 0$.

Passive graph $G^{\star}=G^{\star}\left(n, m, P^{\star}\right)$. In Theorem 2 below we show that a passive graph with an infinite degree variance has the global clustering coefficient $C_{G^{\star}} \approx 1$. By $X$ we denote a random variable with the distribution $\mathrm{P}^{\star}$.

Theorem 2. Let $\beta>0$. Let $m, n \rightarrow \infty$. Assume that $m n^{-1} \rightarrow \beta$ and

(i) $X$ converges in distribution to a random variable $Z$;

(ii) $\mathbf{E} Z^{2}<\infty$ and $\lim _{m, n \rightarrow \infty} \mathbf{E} X^{2}=\mathbf{E} Z^{2}$;

(iii) $\mathbf{E} Z^{3}=\infty$.

Then the clustering coefficient $C_{G^{\star}}=1-o_{P}(1)$.

We mention that under conditions of Theorem 2, the degree $d\left(v_{1}\right)$ converges in distribution to the compound Poisson random variable $d_{*}=\sum_{j=1}^{\zeta} \tilde{Z}_{j}$, see [1]. Here $\tilde{Z}_{1}, \tilde{Z}_{2}, \ldots$ are independent random variables with the common probability distribution $\mathbf{P}\left(\tilde{Z}_{1}=r\right)=(r+1) \mathbf{P}(Z=r+$ 1) $\mathbf{E} Z, r=0,1,2, \ldots$ The random variable $\zeta$ is independent of the sequence $\tilde{Z}_{1}, \tilde{Z}_{2}, \ldots$ and has Poisson distribution with mean $\mathbf{E} \zeta=\beta^{-1} \mathbf{E} Z$. Assuming that for some $\alpha \in(3,4)$ and $c>0$

$$
\mathbf{P}(Z=r)=c r^{-\alpha}(1+o(1)) \quad \text { as } \quad r \rightarrow+\infty,
$$

we obtain, by Theorem 4.30 of [8], that

$$
\mathbf{P}\left(d_{*}=r\right)=\mathbf{P}\left(\tilde{Z}_{1}=r\right)(\mathbf{E} \zeta)(1+o(1))=c^{\prime} \beta^{-1} r^{1-\alpha}(1+o(1)) \quad \text { as } \quad r \rightarrow+\infty,
$$

for some constant $c^{\prime}>0$. In this case $G^{*}$ has asymptotic power law degree distribution with a finire first moment, infinite variance and the clustering coefficient $C_{G^{*}} \approx 1$.

Inhomogeneous graph $G\left(n, m, P_{X}, P_{Y}\right)$. In Theorem 3 below we show that the global clustering coefficient of an inhomogeneous graph with an infinite degree variance is highly determined by the ratio of the random variables

$$
S_{X}=\sum_{i=1}^{m} X_{i}^{3} \quad \text { and } \quad S_{Y}=\sum_{j=1}^{n} Y_{j}^{2} .
$$

We denote $a_{i}=\mathbf{E} X_{1}^{i}, i=1,2$, and $b_{1}=\mathbf{E} Y_{1}$. 
Theorem 3. Let $\beta>0$. Let $m, n \rightarrow \infty$. Assume that $m n^{-1} \rightarrow \beta$. Suppose that $\mathbf{E} X_{1}^{2}<\infty$, $\mathbf{E} X_{1}^{3}=\infty, \mathbf{E} Y_{1}<\infty, \mathbf{E} Y_{1}^{2}=\infty$. Denote $\kappa=\beta^{3 / 2} a_{2}^{2} b_{1}^{-1}$. We have $C_{G}=\left(1+\kappa S_{Y} / S_{X}\right)^{-1}+o_{P}(1)$.

In the case where $S_{X}$ and $S_{Y}$ grow to infinity at the same rate we can obtain a non-trivial limit of $C_{G}$. The next remark addresses the case where the distributions of $X_{1}^{3}$ and $Y_{1}^{2}$ belong to the domain of attraction of stable distributions having the same characteristic exponent $\alpha \leq 1$.

Remark 1. Let $\alpha, \beta>0$. Let $m, n \rightarrow \infty$. Assume that $m n^{-1} \rightarrow \beta$. Suppose that for some $c_{x}, c_{y}>0$ we have

$$
\mathbf{P}\left(X_{1}>t\right)=c_{x} t^{-3 \alpha}+o\left(t^{-3 \alpha}\right), \quad \mathbf{P}\left(Y_{1}>t\right)=c_{y} t^{-2 \alpha}+o\left(t^{-2 \alpha}\right) \quad \text { as } \quad t \rightarrow+\infty .
$$

(i) For $0<\alpha<1$ the ratio $S_{Y} / S_{X}$ converges in distribution to the random variable $c^{*} Z_{\alpha} / Z_{\alpha}^{\prime}$, where $Z_{\alpha}, Z_{\alpha}^{\prime}$ are independent stable random variables with the Laplace transform $\mathbf{E} e^{-s Z_{\alpha}}=$ $\mathbf{E} e^{-s Z_{\alpha}^{\prime}}=e^{-s^{\alpha}}$ and $c^{*}=\left(c_{y} /\left(c_{x} \beta\right)\right)^{1 / \alpha}$.

(ii) For $\alpha=1$ the ratio $S_{Y} / S_{X}=c_{y}\left(c_{x} \beta\right)^{-1}+o_{P}(1)$.

Let us apply Theorem 3 to power law random weights (3). We observe that $\mathbf{E} X_{1}^{2}, \mathbf{E} Y_{1}<\infty$ and $\mathbf{E} X_{1}^{3}, \mathbf{E} Y_{1}^{2}=\infty$ imply $2 / 3<\alpha \leq 1$. For $\alpha=1$ the result of Theorem 3 implies that $C_{G} \approx\left(1+\kappa c_{y} /\left(c_{x} \beta\right)\right)^{-1}$ is asymptotically constant. For $2 / 3<\alpha<1$ it implies that $C_{G}$ converges in distribution to the random variable $\left(1+\kappa\left(c_{y} /\left(c_{x} \beta\right)\right)^{1 / \alpha} Z_{\alpha} / Z_{\alpha}^{\prime}\right)^{-1}$.

Finally, we mention that for $m / n \rightarrow \beta \in(0,+\infty)$ and $2 / 3<\alpha \leq 1$, the inhomogeneous graph defined by power law weights $(3)$ has a power law asymptotic degree distribution with the tail index $3 \alpha-1$, see [2]. In particular, the asymptotic degree distribution has a finite first moment and an infinite variance.

\subsection{Discussion}

One motivation of our study was the recent paper [17, which claims that "if the degree distribution obeys the power law with an infinite variance, then the global clustering coefficient tends to zero with high probability as the size of a graph grows." This may look a bit confusing in view of the fact that some large social networks with quite substantial global clustering coefficients are believed to have a power law degree distribution with an infinite variance. The present study could be viewed as an attempt to resolve this seemingly contradiction with the aid of a known theoretical model of an affiliation network.

We observe that random intersection graphs considered in this paper admit asymptotic power law degree distributions, but their degree sequence is not an iid sample from a power law. We mention that some real affiliation networks are believed to have a power law degree sequence, but with an exponential cutoff, [15], [16], [18].

In what follows we discuss the relation between the result of [17] and our Theorems 1, 2, 3 in some detail. To this aim we briefly recall the argument of [17]. We call a path $x \sim y \sim z$ a cherry produced by vertex $y$. For example, a vertex $v_{j}$ of degree $d_{j}=d\left(v_{j}\right)$ produces $\left(\begin{array}{c}d_{j} \\ 2\end{array}\right)$ cherries. Ostroumova and Samosvat [17] observed that cherries produced by vertices of large degrees highly outnumber the triangles of the graph. Indeed, among the iid degrees $d_{1}, \ldots, d_{n}$ obeying a power law with the tail index $1<\alpha<2$, the largest few roughly scale as $n^{1 / \alpha}$. Consequently, the number of cherries produced by the largest vertices roughly scale as $n^{2 / \alpha}$. On the other hand, the number of triangles incident to any vertex $v_{j}$ does not exceed the number of cherries $\left(\begin{array}{c}d_{j} \\ 2\end{array}\right)$. More importantly, this number is bounded by the total number of edges of the graph (edges needed to close cherries produced by $v_{j}$ ). But for $1<\alpha$ the average degree is 
bounded and the total number of edges scales as $n$. This implies that only a negligible fraction $n^{1-(2 / \alpha)}$ of cherries produced by the largest vertices are closed. Putting things together one can show that $3 \Delta \leq c \sum_{j} n \wedge\left(\begin{array}{c}d_{j} \\ 2\end{array}\right)$ is negligible compared to $\Lambda=\sum_{j}\left(\begin{array}{c}d_{j} \\ 2\end{array}\right)$. Hence $C_{G}=o_{P}(1)$.

In a random intersection graph $G$ the triadic closure of a cherry is explained by a common attribute shared by all three vertices of the cherry (triangles whose edges are witnessed by distinct attributes are rare and can be neglected). We exploit this clustering mechanism while evaluating the global clustering coefficient $C_{G}$ : When counting triangles we focus on cliques of $G$ induced by the neighbourhoods $D_{i}=D_{w_{i}} \subset V$ of attributes $w_{i} \in W$ in the underlying bipartite graph $H$. Every set $D_{i}$ of size $\tilde{X}_{i}:=\left|D_{i}\right|$ covers $\left(\begin{array}{c}\tilde{X}_{i} \\ 3\end{array}\right)$ triangles of $G$ and the total number of triangles obtained in this way scales as $\tilde{S}_{X}=\sum_{i}\left(\begin{array}{c}\tilde{X}_{i} \\ 3\end{array}\right)$ (overlaps can be neglected). In fact, this number dominates the total number of triangles in each of random intersection graphs considered in Theorems 13 ,

In the active graph (with bounded average degree) the random variables $\tilde{X}_{i}$ have the same asymptotic Poisson distribution. Hence $\widetilde{S}_{X}$ scales as $m$. Furthermore, the degrees $\left\{d_{j}\right\}$ of vertices $\left\{v_{j}\right\}$ can be approximated by asymptotically independent Poisson random variables having means $\lambda_{j}=Z_{j} \beta^{-1} \mathbf{E} Z_{j}$. Here $Z_{1}, \ldots, Z_{n}$ are iid copies of $Z$. Hence $\Lambda=\sum_{j}\left(\begin{array}{c}d_{j} \\ 2\end{array}\right)$ scales as $\Theta\left(S_{Z}\right)$, where $S_{Z}=\sum_{j} Z_{j}^{2}$. For $\mathbf{E} Z^{2}=\infty$ the sum $S_{Z}$ is super-linear in $n$ and for $n=\Theta(m)$ we obtain $\tilde{S}_{X} / S_{Z}=o_{P}(1)$. Thus $C_{G}=o_{P}(1)$. We note that similarly to the case of iid degrees considered in [17] the number of cherries of active intersection graph scales as a sum of iid random variables having an infinite mean. One difference from [17] is that in our Theorem 1 we have relaxed the structural "power law degree" condition of [17].

The passive graph is a union of independently located cliques induced by the sets $D_{w_{i}} \subset V$, $w_{i} \in W$. Since $\left|D_{w_{i}}\right|=\tilde{X}_{i}$ converges in distribution to a random variable having infinite third moment, we have that $\tilde{S}_{X}$ is super-linear in $m$. Furthermore, we show that $\Lambda$ is dominated by the number of cherries covered by the cliques. This number scales as $3 \sum_{i}\left(\begin{array}{c}X_{i} \\ 3\end{array}\right)=3 \tilde{S}_{X}$ (we neglect overlaps again). Hence, $C_{G}^{*}=1+o_{P}(1)$.

The inhomogeneous graph interpolates between the active and passive graphs. The number of triangles $\tilde{S}_{X}$ scales as $\Theta\left(S_{X}\right)$ as in the passive graph, while $\Lambda$ is approximately the sum of the number of cherries covered by large cliques (as in the passive graph) and the number of cherries produced by the largest vertices (as in the active graph). These numbers scale as $3 \tilde{S}_{X}$ and $\Theta\left(S_{Y}\right)$ respectively. In this way we obtain the approximation $C_{G} \approx\left(1+\Theta\left(S_{Y} / S_{X}\right)\right)^{-1}$. Finally, we note that the inhomogeneous graph is a fitness model of a real affiliation network, where activity of vertices is modeled by the distribution $P_{Y}$ and attractiveness of attributes is modeled by the distribution $P_{X}$. We summarize the result of Theorem 3 as follows: The global clustering coefficient is non-vanishing whenever the attractiveness "outweighs" the activity.

\section{Proofs}

We begin by establishing some notation. Detailed proofs are given afterwards.

Notation. By $\mathbf{E}_{\mathbb{X}}$ and $\mathbf{P}_{\mathbb{X}}$ (respectively $\tilde{\mathbf{E}}$ and $\tilde{\mathbf{P}}$ ) we denote the conditional expectation and conditional probability given $\mathbb{X}=\left(X_{1}, \ldots, X_{m}\right)$ (respectively $\mathbb{X}$ and $\mathbb{Y}=\left(Y_{1}, \ldots, Y_{n}\right)$ ). We use the notation $[k]$ for the set $\{1,2, \ldots, k\}$ and the shorthand notation $\sum_{\Lambda}$ for the double sum $\sum_{x \in V} \sum_{\{y, z\} \subset V \backslash\{x\}}$. Denote empirical means $\hat{a}_{r}=m^{-1} \sum_{i \in[m]} X_{i}^{r}$ and $\hat{b}_{r}=n^{-1} \sum_{j \in[n]} Y_{j}^{r}$.

Let $G$ be the intersection graph defined by a bipartite graph $H$ with the bipartition $V \cup W$. For $x, y \in V$ and $w \in W$ we denote by $\mathbb{I}_{x \sim y}$ and $\mathbb{I}_{x w}$ the indicators of the events that $x, y$ are adjacent in $G$ and $x, w$ are adjacent in $H$. For $v=v_{j} \in V$ and $w=w_{i} \in W$ we write interchangeably $Y_{j}$ or $Y_{v}$ and $X_{i}$ or $X_{w}$ also $p_{i j}$ or $p_{w v}$. For $v \in V$ and $w \in W$ we denote $\lambda_{v w}=Y_{v} X_{w}(n m)^{-1 / 2}$. 
For $w \in W$, let $D_{w} \subset V$ denote the set of neighbours of $w$ in $H$. Note that each $D_{w}$ induces a clique in $G$. Given a subgraph $G^{\prime} \subset G$ and a subset $W^{\prime} \subset W$ we say that the collection of sets $\left\{D_{w}, w \in W^{\prime}\right\}$ is a cover of $G^{\prime}$ if every edge of $G^{\prime}$ is witnessed by some $w \in W^{\prime}$ and for every $w \in W^{\prime}$ there is an edge in $G^{\prime}$ having no other witness from $W^{\prime}$, but $w$ (any proper subset of $W^{\prime}$ can't be a cover of $G^{\prime}$ ).

A subgraph of $G$ is labeled "lucky" if it has a cover consisting of a single set $D_{w}$, for some $w \in W$. A subgraph is labeled "unlucky" if it has a cover consisting of two or more sets. We note that a subgraph can be labeled "lucky" and "unlucky" simultaneously.

The numbers of lucky and unlucky triangles (2-paths) are denoted by $\Delta_{L}$ and $\Delta_{U}\left(\Lambda_{L}\right.$ and $\left.\Lambda_{U}\right)$. The number of triangles (2-paths) receiving both lucky and unlucky labels is denoted $\Delta_{L U}$ $\left(\Lambda_{L U}\right)$. Clearly, we have

$$
\Delta=\Delta_{L}+\Delta_{U}-\Delta_{L U}, \quad \Lambda=\Lambda_{L}+\Lambda_{U}-\Lambda_{L U} .
$$

Proof of Theorem [1. In the proof we use some ideas of [17]. Before the proof we collect notation and auxiliary facts. Let $Z_{1}, Z_{2}, \ldots$ be iid copies of $Z$. We denote by $\mathbf{E}_{Z}\left(\operatorname{Var}_{Z}\right)$ the conditional expectation (variance) given the sequence $\left\{Z_{i}, i \geq 1\right\}$. Furthermore, we denote $z_{1}=\mathbf{E} Z$ and $S_{Z}=\sum_{i \in[n]} Z_{i}^{2}$. Given $A \subset[n]$ we denote $S_{Z, A}=\sum_{i \in A} Z_{i}^{2}$. By $d_{i, A}=\sum_{j \in A \backslash\{i\}} \mathbb{I}_{v_{i} \sim v_{j}}$ we denote the number of neighbours from the set $\left\{v_{j}, j \in A\right\} \subset V$ of a vertex $v_{i}$ in the intersection graph $G$. In the proof we use the following inequalities for the intersection probability of two independent uniformly distributed random subsets $\mathcal{S}, \mathcal{T} \subset W$ (see, e.g., Lemma 6 of [1])

$$
s t m^{-1}(1-s t /(m-s)) \leq \mathbf{P}(\mathcal{S} \cap \mathcal{T} \neq \emptyset|| \mathcal{S}|=s,| \mathcal{T} \mid=t) \leq s t m^{-1}
$$

We recall that every vertex $v_{i} \in V=\left\{v_{1}, \ldots, v_{n}\right\}$ is prescribed a subset $S_{i} \subset W=\left\{w_{1}, \ldots, w_{m}\right\}$ of size $\left|S_{i}\right|=\min \left\{m, Z_{i}\right\}$. Furthermore, the condition $\mathbf{E} Z<\infty$ ensures the existence of a positive sequence $\varepsilon_{n} \downarrow 0$ such that

$$
\mathbf{P}\left(\max _{i \in[n]} Z_{i}<n \varepsilon_{n}\right)=1-o(1)
$$

see Lemma 3. Note that (6) implies $\mathbf{P}\left(\max _{i \in[n]} Z_{i}<m\right)=1-o(1)$.

Now we prove the theorem. For this purpose we show that there is a constant $c^{*}>0$ and a sequence $\varkappa_{n} \downarrow 0$ both depending on the distribution of $Z$ and on $\beta$ such that

$$
\begin{aligned}
& \mathbf{P}\left(\Lambda>c^{*} S_{Z}\right)=1-o(1), \\
& \mathbf{P}\left(\Delta \leq n^{3 / 2} \varkappa_{n}\right)=1-o(1), \\
& \Delta=O_{P}\left(n+n^{-3} S_{Z}^{3}\right) .
\end{aligned}
$$

Let us show that (7), (8), (9) imply $C_{G}=o_{P}(1)$. Introduce the event $B=\left\{S_{Z} \leq n^{3 / 2} \sqrt{\varkappa_{n}}\right\}$ and let $\bar{B}$ denote the complement event. We have

$$
C_{G}=\frac{3 \Delta}{\Lambda}=\frac{3 \Delta}{\Lambda} \mathbb{I}_{B}+\frac{3 \Delta}{\Lambda} \mathbb{I}_{\bar{B}}=O_{P}\left(\frac{n}{S_{Z}}\right)+O_{P}\left(\varkappa_{n}\right)+O_{P}\left(\sqrt{\varkappa_{n}}\right)=o_{P}(1) .
$$

Here on the event $B$ we have bounded $\Delta$ using $(9)$ and on the event $\bar{B}$ we have applied $(8)$. In the final step we invoked the bound $n / S_{Z}=o_{P}(1)$, which follows by Lemma 1 . It remains to prove (7), (8) and (9).

Proof of (7). Fix $0<a<b$ such that $p:=\mathbf{P}(a<Z<b)>0$. Define random subsets of $[n]$

$$
R=\left\{i: a<Z_{i}<b\right\}, \quad T=\left\{i: Z_{i} \leq \ln ^{2} n\right\}, \quad \Theta=\left\{i: \ln ^{2} n<Z_{i} \leq n \varepsilon_{n}\right\} .
$$


Note that for any $i \in[n]$ and $A \subset[n]$ the degree $d_{i}$ of a vertex $v_{i}$ is larger or equal to $d_{i, A}$. Therefore, we have

$$
\Lambda=\sum_{i \in[n]}\left(\begin{array}{c}
d_{i} \\
2
\end{array}\right) \geq \Lambda_{T}+\Lambda_{\Theta}, \quad \Lambda_{T}=\sum_{i \in T}\left(\begin{array}{c}
d_{i, T} \\
2
\end{array}\right), \quad \Lambda_{\Theta}=\sum_{i \in \Theta}\left(\begin{array}{c}
d_{i, R} \\
2
\end{array}\right) .
$$

In order to prove (7) we show below that

$$
\Lambda_{T}=\left(1+o_{P}(1)\right) 2^{-1} \beta^{-2} z_{1}^{2} S_{Z, T}, \quad \mathbf{P}\left(\Lambda_{\Theta} \geq\left(\frac{a p}{4 \beta}\right)^{2} S_{Z, \Theta}\right)=1-o(1) .
$$

Indeed, (11), 12 combined with the identity $S_{Z, T}+S_{Z, \Theta}=S_{Z}$, which holds with probability $1-o(1)$ (see (6)), imply (7).

Proof of the first relation of $(12)$. In view of Lemma 2 it suffices to show that

$$
\mathbf{E}_{Z} \Lambda_{T}=\left(1+o_{P}(1)\right) 2^{-1} \beta^{-2} z_{1}^{2} S_{Z, T}, \quad \operatorname{Var}_{Z} \Lambda_{T}=o_{P}\left(S_{Z, T}^{2}\right)
$$

We note that the sum $S_{Z, T}=\sum_{i \in[n]} Z_{i}^{2} \mathbb{I}_{Z_{i}<\ln ^{2} n}$ is superlinear in $n$ as $n \rightarrow+\infty$, see Lemma 1 . To prove the first relation of (13) we write

$$
\Lambda_{T}=\sum_{i \in T} \sum_{\{j, k\} \subset T \backslash\{i\}} \mathbb{I}_{v_{i} \sim v_{j}} \mathbb{I}_{v_{i} \sim v_{k}}
$$

and evaluate the expectation

$$
\mathbf{E}_{Z} \Lambda_{T}=\sum_{i \in T} \sum_{\{j, k\} \subset T \backslash\{i\}} \bar{p}_{i j} \bar{p}_{i k}, \quad \bar{p}_{i j}:=\mathbf{P}_{Z}\left(v_{i} \sim v_{j}\right)=\mathbf{P}_{Z}\left(S_{i} \cap S_{j} \neq \emptyset\right) .
$$

Invoking the inequalities that follow from (5)

$$
Z_{i} Z_{j} m^{-1}\left(1-2 m^{-1} \ln ^{4} n\right) \leq \bar{p}_{i j} \leq Z_{i} Z_{j} m^{-1}
$$

we obtain

$$
\mathbf{E}_{Z} \Lambda_{T}=\left(1+O\left(\frac{\ln ^{4} n}{m}\right)\right) \sum_{i \in T} \sum_{\{j, k\} \subset T \backslash\{i\}} \frac{Z_{i}^{2} Z_{j} Z_{k}}{m^{2}}=\left(1+o_{P}(1)\right) S_{Z, T} \frac{1}{2} \frac{\hat{z}_{1, T}^{2}}{\beta^{2}} .
$$

Here we denote $\hat{z}_{1, T}:=n^{-1} \sum_{i \in T} Z_{i}$. Finally, the law of large numbers implies $\hat{z}_{1, T}=z_{1}+o_{P}(1)$. To prove the second relation of (13) we write $\Lambda_{T}$ in the form $\Lambda_{T}=\mathbf{E}_{Z} \Lambda_{T}+L_{T}+Q_{T}$, where

$$
\begin{aligned}
L_{T} & =\sum_{\{i, j\} \subset T}\left(\mathbb{I}_{v_{i} \sim v_{j}}-\bar{p}_{i j}\right) \sum_{k \in T \backslash\{i, j\}}\left(\bar{p}_{i k}+\bar{p}_{j k}\right), \\
Q_{T} & =\sum_{i \in T} \sum_{\{j, k\} \subset T \backslash\{i\}}\left(\mathbb{I}_{v_{i} \sim v_{j}}-\bar{p}_{i j}\right)\left(\mathbb{I}_{v_{i} \sim v_{k}}-\bar{p}_{i k}\right) .
\end{aligned}
$$

We observe that $L_{T}$ and $Q_{T}$ are conditionally uncorrelated (given $\left\{Z_{n}\right\}$ ). Therefore

$$
\operatorname{Var}_{Z} \Lambda_{T}=\operatorname{Var}_{Z} L_{T}+\operatorname{Var}_{Z} Q_{T}
$$


We bound the summands on the right using (14). A simple calculation shows that

$$
\begin{aligned}
\operatorname{Var}_{Z} L_{T} & =\sum_{\{i, j\} \subset T} \bar{p}_{i j}\left(1-\bar{p}_{i j}\right)\left(\sum_{k \in T \backslash\{i, j\}}\left(\bar{p}_{i k}+\bar{p}_{j k}\right)\right)^{2} \\
& \leq \sum_{\{i, j\} \subset T} \frac{Z_{i} Z_{j}}{m}\left(Z_{i} \beta^{-1} \hat{z}_{1, T}+Z_{j} \beta^{-1} \hat{z}_{1, T}\right)^{2} \\
& \leq 2 \beta^{-3} \hat{z}_{1, T}^{3} \sum_{i \in T} Z_{i}^{3} .
\end{aligned}
$$

Now, invoking the inequality $\sum_{i \in T} Z_{i}^{3} \leq S_{Z, T} \max _{i \in T} Z_{i} \leq S_{Z, T}^{3 / 2}$ and the bound $\hat{z}_{1, T}=O_{P}(1)$ we obtain $\operatorname{Var}_{Z} L_{T}=O_{P}\left(S_{Z, T}^{3 / 2}\right)=o_{P}\left(S_{Z, T}^{2}\right)$. Furthermore, we have

$$
\operatorname{Var}_{Z} Q_{T}=\sum_{i \in T} \sum_{\{j, k\} \subset T \backslash\{i\}} \bar{p}_{i j}\left(1-\bar{p}_{i j}\right) \bar{p}_{i k}\left(1-\bar{p}_{i k}\right) \leq \sum_{i \in T} \sum_{\{j, k\} \subset T \backslash\{i\}} \bar{p}_{i j} \bar{p}_{i k}
$$

Invoking the inequality $\bar{p}_{i j} \bar{p}_{i k} \leq Z_{i}^{2} Z_{j} Z_{k} m^{-2}$ (which follows from (14)) we obtain

$$
\operatorname{Var}_{Z} Q_{T} \leq S_{Z, T} \hat{z}_{1, T}^{2} \beta^{-2}=O_{P}\left(S_{Z, T}\right)=o_{P}\left(S_{Z, T}^{2}\right)
$$

Finally, 15 implies $\operatorname{Var}_{Z} \Lambda_{T}=o_{P}\left(S_{Z, T}^{2}\right)$.

Proof of the second relation of (12). For every $i \in \Theta$ and $j \in R$ we have, by (5),

$$
\mathbf{P}_{Z}\left(v_{i} \sim v_{j}\right) \geq 0.9 a m^{-1} Z_{i}=: q_{i} .
$$

Here 0.9 is a lower bound for the number $1-Z_{i} Z_{j} /\left(m-Z_{j}\right)$ valid for sufficiently large $m, n$. We note that conditionally, given $\left\{Z_{i}, i \geq 1\right\}$ and $|R|$, the random variable $d_{i, R}$ is a sum of independent indicators (their number is $|R|$ ) each having success probability at least $q_{i}$. Furthermore, $|R|$ has binomial distribution with mean $n p$. Given $t \geq 0$ we have

$$
\mathbf{P}\left(d_{i, R} \geq t\right) \geq \mathbf{P}\left(d_{i, R} \geq t|| R \mid \geq n p / 2\right)-r_{1} \geq \mathbf{P}\left(L_{i} \geq t\right)-r_{1} .
$$

Here $r_{1}=\mathbf{P}(|R|<n p / 2)$ and $L$ is the sum of $n^{\prime}:=\lfloor n p / 2\rfloor$ independent indicators with the same success probability $q_{i}$. Chernoff's inequality implies

$$
\mathbf{P}(|R|<n p / 2) \leq e^{-n p / 4}=O\left(n^{-9}\right), \quad \mathbf{P}\left(L<n^{\prime} q_{i} / 2\right) \geq e^{-n^{\prime} q_{i} / 4}=O\left(n^{-9}\right) .
$$

Note that the second bound holds uniformly in $i \in \Theta$, since $Z_{i} \geq \ln ^{2} n$ for $i \in \Theta$. Choosing $t_{i}=n^{\prime} q_{i} / 2$ in (16) we obtain

$$
\mathbf{P}\left(d_{i, R} \geq t_{i}, i \in \Theta\right) \geq 1-O\left(n^{-8}\right) .
$$

This bound implies the second relation of 12 .

Proof of (9). We recall that $\max _{i \in[n]} Z_{i} \leq m$ with probability $1-o(1)$. Assuming that this inequality holds we prove below that $\mathbf{E}_{Z} \Delta \leq O_{P}\left(n+n^{-3} S_{Z}^{3}\right)$. Now 9 follows by Lemma 2 (ii). We have $\Delta \leq \Delta_{L}+\Delta_{U}$, where the numbers $\Delta_{L}$ and $\Delta_{U}$ of lucky and unlucky triangles satisfy

$$
\begin{aligned}
\Delta_{L} & \leq \sum_{w \in W} \sum_{\{i, j, k\} \subset[n]} \mathbb{I}_{w \in S_{i}} \mathbb{I}_{w \in S_{j}} \mathbb{I}_{w \in S_{k}}, \\
\Delta_{U} & \leq \sum_{\substack{w, \tau, \varkappa \in W \\
w \neq \tau \neq \varkappa}} \sum_{\{i, j, k\} \subset[n]} \mathbb{I}_{w \in S_{i}} \mathbb{I}_{w \in S_{j}} \mathbb{I}_{\tau \in S_{i}} \mathbb{I}_{\tau \in S_{k}} \mathbb{I}_{\varkappa \in S_{j}} \mathbb{I}_{\varkappa \in S_{k}} .
\end{aligned}
$$


Invoking the identity $\mathbf{P}_{Z}\left(w \in S_{i}\right)=m^{-1} Z_{i}$ and inequality $\mathbf{P}_{Z}\left(w, \tau \in S_{i}\right) \leq m^{-2} Z_{i}^{2}$ we obtain

$$
\begin{aligned}
& \mathbf{E}_{Z} \Delta_{L} \leq m^{-2} \sum_{\{i, j, k\} \subset[n]} Z_{i} Z_{j} Z_{k} \leq \beta^{-2} \hat{z}_{1}^{3} n=O_{P}(n), \\
& \mathbf{E}_{Z} \Delta_{U} \leq m^{-3} \sum_{\{i, j, k\} \subset[n]} Z_{i}^{2} Z_{j}^{2} Z_{k}^{2} \leq \beta^{-3} n^{-3} S_{Z}^{3} .
\end{aligned}
$$

Proof of (8). By Lemma 3, we can find an increasing positive function $\psi(t) \uparrow+\infty$ as $t \rightarrow+\infty$ such that $\mathbf{E} Z \psi(Z)<\infty$. We can assume that $\psi(t)<t^{1 / 4}$, for $t \geq 1$. Denote $\delta_{n}=1 / \psi\left(n^{1 / 4}\right)$ and $\tau_{n}=\mathbf{E} Z \psi(Z) \mathbb{I}_{\left\{Z \geq n^{1 / 4}\right\}}$. Put $\varkappa_{n}=\min \left\{\delta_{n}^{1 / 4}, \tau_{n}^{1 / 4}\right\}$. Clearly, $\delta_{n} \downarrow 0, \tau_{n} \downarrow 0$ and $\varkappa_{n} \downarrow 0$. We observe that

$$
\mathbf{E} Z^{2} \mathbb{I}_{\left\{Z<\sqrt{n} \delta_{n}\right\}} \leq \sqrt{n} \delta_{n} z_{1}, \quad \mathbf{P}\left(Z \geq \sqrt{n} \delta_{n}\right) \leq \frac{\mathbf{E} Z \psi(Z) \mathbb{I}_{\left\{Z \geq \sqrt{n} \delta_{n}\right\}}}{\sqrt{n} \delta_{n} \psi\left(\sqrt{n} \delta_{n}\right)} \leq \frac{\tau_{n}}{\sqrt{n}} .
$$

Now we estimate $\Delta$. We observe that the number $\Delta_{i}$ of triangles incident to a given vertex $v_{i} \in V$ is at most $\left(\begin{array}{c}d_{i} \\ 2\end{array}\right)$. Furthermore, $\Delta_{i}$ is always less than the total number of edges in the graph, denoted by $\mathcal{E}$. Therefore, we have

$$
3 \Delta=\sum_{i \in[n]} \Delta_{i} \leq \sum_{i \in[n]: Z_{i}<\sqrt{n} \delta_{n}}\left(\begin{array}{c}
d_{i} \\
2
\end{array}\right)+\mathcal{E} \sum_{i \in[n]: Z_{i} \geq \sqrt{n} \delta_{n}} 1=: U_{1}+\mathcal{E} U_{2} .
$$

We show below that $\mathbf{E} U_{1}=O\left(n^{3 / 2} \delta_{n}\right), \mathbf{E} U_{2}=O\left(\sqrt{n} \tau_{n}\right)$ and $\mathbf{E} \mathcal{E}=O(n)$. These bounds together with (19) imply (8).

For $\mathcal{E}=\sum_{\{u, v\} \subset V} \mathbb{I}_{u \sim v}$ we have, by (5),

$$
\mathbf{E} \mathcal{E}=\left(\begin{array}{l}
n \\
2
\end{array}\right) \mathbf{P}\left(v_{1} \sim v_{2}\right) \leq\left(\begin{array}{l}
n \\
2
\end{array}\right) \mathbf{E}\left(Z_{1} Z_{2} / m\right) \leq \frac{n^{2}}{2 m}\left(\mathbf{E} Z_{1}\right)^{2}=O(n) .
$$

For $U_{2}=\sum_{i \in[n]} \mathbb{I}_{Z_{i}>\sqrt{n} \delta_{n}}$ we have, see (18),

$$
\mathbf{E} U_{2} \leq n \mathbf{P}\left(Z_{1}>\sqrt{n} \delta_{n}\right) \leq \sqrt{n} \tau_{n} .
$$

It remains to bound $\mathbf{E} U_{1}$. For every $i$ we have, by (5),

$$
\mathbf{E}\left(\left(\begin{array}{c}
d_{i} \\
2
\end{array}\right) \mid Z_{i}\right)=\sum_{\{k, r\} \subset[n] \backslash\{i\}} \mathbf{P}\left(v_{k} \sim v_{i} \mid Z_{i}\right) \mathbf{P}\left(v_{r} \sim v_{i} \mid Z_{i}\right) \leq\left(\begin{array}{c}
n-1 \\
2
\end{array}\right) Z_{i}^{2}\left(\mathbf{E} Z_{1}\right)^{2} m^{-2} .
$$

Invoking the first inequality of 18 we obtain

$$
\mathbf{E}\left(\begin{array}{c}
d_{i} \\
2
\end{array}\right) \mathbb{I}_{\left\{Z_{i} \leq \sqrt{n} \delta_{n}\right\}} \leq\left(\begin{array}{c}
n-1 \\
2
\end{array}\right) \frac{z_{1}^{2}}{m^{2}} \mathbf{E} Z_{i}^{2} \mathbb{I}_{\left\{Z_{i} \leq \sqrt{n} \delta_{n}\right\}} \leq \frac{z_{1}^{3}}{2 \beta^{2}} \sqrt{n} \delta_{n} .
$$

Finally, we have

$$
\mathbf{E} U_{1}=\sum_{i \in[n]}\left(\begin{array}{c}
d_{i} \\
2
\end{array}\right) \mathbb{I}_{Z_{i} \leq \sqrt{n} \delta_{n}} \leq \frac{z_{1}^{3}}{2 \beta^{2}} n^{3 / 2} \delta_{n}
$$


Proof of Theorem 2. In the proof we use the notation $X_{i}=\left|D_{w_{i}}\right|, w_{i} \in W$, and $S_{X}=\sum_{i \in[n]} X_{i}^{3}$. We firstly count triangles. For every $w \in W$ there are $N_{w}:=\left(\begin{array}{c}\left|D_{w}\right| \\ 3\end{array}\right)$ lucky triangles covered by $D_{w}$. We have, by inclusion-exclusion, that

$$
N-N^{*} \leq \Delta_{L} \leq N, \quad \text { where } \quad N=\sum_{w \in W}\left(\begin{array}{c}
\left|D_{w}\right| \\
3
\end{array}\right), \quad N^{*}=\sum_{\{w, \tau\} \subset W}\left(\begin{array}{c}
\left|D_{w} \cap D_{\tau}\right| \\
3
\end{array}\right) .
$$

Here $\left(\begin{array}{c}\left|D_{w} \cap D_{\tau}\right| \\ 3\end{array}\right)$ counts triangles covered by $D_{w}$ and $D_{\tau}$ simultaneously. Every unlucky triangle has its edges covered by distinct sets. Therefore, $\Delta_{U}$ is at most the sum

$$
N^{* *}:=\sum_{\{x, y, z\} \subset V} \sum_{1 \leq i \neq j \neq k \leq m} \mathbb{I}_{\{x, y\} \subset D_{i}} \mathbb{I}_{\{x, z\} \subset D_{j}} \mathbb{I}_{\{y, z\} \subset D_{k}} .
$$

We estimate the total number of triangles $\Delta$ from the inequalities $\Delta_{L} \leq \Delta \leq \Delta_{L}+\Delta_{U}$. Hence

$$
|\Delta-N| \leq N^{*}+N^{* *} .
$$

We secondly count 2-paths. We have $\Lambda=\Lambda_{L}+\Lambda_{U}-\Lambda_{L U}$, where $\Lambda_{L U}$ is the number of paths labeled both lucky and unlucky. For the number of lucky paths $\Lambda_{L}=3 \Delta_{L}$, we can evaluate $\Lambda_{L}$ using (21). Furthermore, the number $\Lambda_{U}$ of unlucky paths is at most the sum

$$
M^{*}:=\sum_{\{w, \tau\} \subset W}\left|D_{w} \cap D_{\tau}\right| \times\left|D_{w}\right| \times\left|D_{\tau}\right| .
$$

Here $\left|D_{w} \cap D_{\tau}\right| \times\left|D_{w}\right| \times\left|D_{\tau}\right|$ is an upper bound for the number of 2-paths with the central vertex belonging to $D_{w} \cap D_{\tau}$ and with the endpoints belonging to $D_{w} \backslash D_{\tau}$ and $D_{\tau} \backslash D_{w}$ respectively. From the inequalities $\Lambda_{L} \leq \Lambda \leq \Lambda_{L}+\Lambda_{U}$ we obtain

$$
|\Lambda-3 N| \leq 3 N^{*}+M^{*} .
$$

Finally, we derive the relation $C_{G^{*}}=3 \Delta / \Lambda=1+o_{P}(1)$ from (22), 23) and the bounds $N^{*}, N^{* *}, M^{*}=o_{P}(N)$ shown below.

Let us bound $N^{*}, N^{* *}, M^{*}$. We note that the sum $S_{X}$ is superlinear in $m$. Indeed, Lemma 1 implies that $\mathbf{P}\left(S_{X}>m \phi_{m}\right)=1+o(1)$ for some $\phi_{m} \uparrow+\infty$. A simple consequence of this fact is that $6 N=\left(1+o_{P}(1)\right) S_{X}$ is superlinear in $m$ as well. Furthermore, the bounds $N^{*}, N^{* *}, M^{*}=$ $o_{P}(N)$ are equivelaent to the bounds $N^{*}, N^{* *}, M^{*}=o_{P}\left(S_{X}\right)$. In order to show these we prove that

$$
\mathbf{E}_{\mathbb{X}} N^{*}=o_{P}\left(S_{X}\right), \quad \mathbf{E}_{\mathbb{X}} N^{* *}=o_{P}\left(S_{X}\right), \quad \mathbf{E}_{\mathbb{X}} M^{*}=o_{P}\left(S_{X}\right),
$$

and apply Lemma 2. To prove the first bound of 24 we write $\left(\begin{array}{c}\left|D_{w} \cap D_{\tau}\right| \\ 3\end{array}\right)$ in the form

$$
\left(\begin{array}{c}
\left|D_{w} \cap D_{\tau}\right| \\
3
\end{array}\right)=\sum_{\{x, y, z\} \subset V} \mathbb{I}_{\{x, y, z\} \subset D_{w}} \mathbb{I}_{\{x, y, z\} \subset D_{\tau}},
$$

evaluate the conditional expectation

$$
\mathbf{E}_{\mathbb{X}} N^{*}=\left(\begin{array}{c}
n \\
3
\end{array}\right) \sum_{\{i, j\} \subset[m]}\left(\begin{array}{c}
X_{i} \\
3
\end{array}\right)\left(\begin{array}{c}
X_{j} \\
3
\end{array}\right)\left(\begin{array}{c}
n \\
3
\end{array}\right)^{-2},
$$

and invoke (37) of Lemma 4. To prove the second bound of $(24)$ we evaluate

$$
\mathbf{E}_{\mathbb{X}} N^{* *}=\left(\begin{array}{c}
n \\
3
\end{array}\right) \sum_{1 \leq i \neq j \neq k \leq m}\left(\begin{array}{c}
X_{i} \\
2
\end{array}\right)\left(\begin{array}{c}
X_{j} \\
2
\end{array}\right)\left(\begin{array}{c}
X_{k} \\
2
\end{array}\right)\left(\begin{array}{c}
n \\
2
\end{array}\right)^{-3}
$$


and invoke (38) Lemma 4. To prove the third bound of (24) we evaluate

$$
\mathbf{E}_{\mathbb{X}} M^{*}=\mathbf{E}_{\mathbb{X}} \sum_{\{i, j\} \subset[m]} X_{i} X_{j} \sum_{x \in V} \mathbb{I}_{x \in D_{i}} \mathbb{I}_{x \in D_{j}}=n^{-1} \sum_{\{i, j\} \subset[m]} X_{i}^{2} X_{j}^{2}
$$

and invoke $(39)$ of Lemma 4 .

Proof of Theorem 3. Before the proof we introduce some notation. We fix positive sequences $\varepsilon \downarrow 0$ and $t_{n} \uparrow+\infty$ such that $\mathbf{P}\left(\max _{i \in[n]} Y_{i}<\varepsilon_{n} t_{n}^{-1} n\right)=1-o(1)$, see Lemma 3. Note that $\mathbf{E} Y_{1} \mathbb{I}_{Y_{1} \geq t_{n}}=o(1)$ implies $n^{-1} \sum_{i \in[n]} Y_{i} \mathbb{I}_{Y_{i} \geq t_{n}}=o_{P}(1)$. We recall that the inhomogeneous graph $G$ is defined by a bipartite graph $H$ with the bipartition $V \cup W$. We color vertices in $V$ white and those in $W$ black. Given a bipartite graph $H^{\prime}=\left(V^{\prime}, W^{\prime} ; E^{\prime}\right)$ with the bipartition $V^{\prime} \cup W^{\prime}$ and the edge set $E^{\prime}$, we color vertices in $V^{\prime}$ white and those in $W^{\prime}$ black. Define the bipartite graphs

$$
\begin{aligned}
& H_{1}=(\{1,2,3\},\{a\} ;\{\{1, a\},\{2, a\},\{3, a\}\}), \\
& H_{2}=(\{1,2,3\},\{a, b\} ;\{\{1, a\},\{2, a\},\{2, b\},\{3, b\}\}), \\
& H_{3}=(\{1,2,3\},\{a, b, c\} ;\{\{1, a\},\{2, a\},\{2, b\},\{3, b\},\{1, c\},\{3, c\}\}), \\
& H_{4}=(\{1,2,3\},\{a, b, c\} ;\{\{1, a\},\{2, a\},\{2, b\},\{3, b\},\{1, c\},\{2, c\}\}), \\
& H_{5}=(\{1,2,3\},\{a, b\} ;\{\{1, a\},\{2, a\},\{3, a\},\{1, b\},\{2, b\}\}) .
\end{aligned}
$$

For $1 \leq i \leq 5$ we denote by $\mathcal{H}_{i}$ the set of copies of $H_{i}$ in $H$. The number of copies is denoted $N_{i}=\left|\mathcal{H}_{i}\right|$. We note that every $H^{\prime} \in \mathcal{H}_{1}$ defines a lucky triangle in $G, H^{\prime \prime} \in \mathcal{H}_{2}$ defines an unlucky path in $G$, and $H^{\prime \prime \prime} \in \mathcal{H}_{3}$ defines an unlucky triangle in $G$. In particular, we have $\Delta_{L} \leq N_{1}, \Lambda_{L} \leq 3 N_{1}, \Delta_{U} \leq N_{3}$, and $\Lambda_{U} \leq N_{2}$. We call an edge $v_{i} \sim v_{j}$ of $G$ heavy if $Y_{i} Y_{j}>\varepsilon_{n} n$. A subgraph of $G$ is called heavy if it contains a heavy edge. Otherwise it is called light. The number of heavy (light) copies of $H_{i}$ is denoted $N_{i}^{+}\left(N_{i}^{-}\right)$.

The theorem follows from (4) and the relations

$$
\begin{aligned}
N_{1}= & \left(1+o_{P}(1)\right) \tilde{\mathbf{E}} N_{1}=6^{-1} \beta^{-3 / 2} b_{1}^{3} S_{X}+o_{P}\left(S_{X}\right), \\
N_{2}= & \left(1+o_{P}(1)\right) \tilde{\mathbf{E}} N_{2}=2^{-1} a_{2}^{2} b_{1}^{2} S_{Y}+o_{P}\left(S_{Y}\right), \\
& \Delta_{L}=N_{1}+o_{P}\left(S_{X}\right), \\
& \Delta_{U}=o_{P}\left(S_{Y}\right), \\
& \Lambda_{U}=N_{2}+o_{P}\left(S_{Y}\right)+o_{P}\left(S_{X}\right), \\
& \Lambda_{L U}=o_{P}\left(S_{X}\right)+o_{P}\left(S_{Y}\right) .
\end{aligned}
$$

Relations (25), 26) follow from Lemmas 2, 5, 6, It remains to prove 27, 270).

We begin with establishing auxiliary facts. Denote

$$
L_{n}:=n^{-1} \sum_{\{i, j\} \subset[n]} Y_{i} Y_{j}\left(Y_{i}+Y_{j}\right) \mathbb{I}_{Y_{i} Y_{j}>\varepsilon_{n} n}, \quad L_{n}^{\prime}:=n^{-1} \sum_{\{i, j\} \subset[n]} Y_{i} Y_{j} \mathbb{I}_{Y_{i} Y_{j}>\varepsilon_{n} n} .
$$

We have

$$
\begin{array}{ll}
L_{n}=o_{P}\left(S_{Y}\right), & L_{n}^{\prime}=o_{P}(n) \\
N_{1}^{+}=o_{P}\left(S_{X}\right), & N_{2}^{+}=o_{P}\left(S_{Y}\right), \\
N_{3}^{-}=o_{P}\left(S_{Y}\right), & N_{4}^{-}=o_{P}\left(S_{Y}\right), \quad N_{5}^{-}=o_{P}\left(S_{X}\right) .
\end{array}
$$


Proof of 31 . On the event $\left\{\max _{i \in[n]} Y_{i} \leq \varepsilon_{n} t_{n}^{-1} n\right\}$ which has probability $1-o(1)$ we have

$$
Y_{i} Y_{j}\left(Y_{i}+Y_{j}\right) \mathbb{I}_{Y_{i} Y_{j}>\varepsilon_{n} n} \leq Y_{i}^{2} Y_{j} \mathbb{I}_{Y_{j}>t_{n}}+Y_{j}^{2} Y_{i} \mathbb{I}_{Y_{i}>t_{n}}
$$

Hence $L_{n} \leq S_{Y} n^{-1} \sum_{i \in[n]} Y_{i} \mathbb{I}_{Y_{i}>t_{n}}$. The bound $n^{-1} \sum_{i \in[n]} Y_{i} \mathbb{I}_{Y_{i}>t_{n}}=o_{P}(1)$ implies the first bound of (31). The second bound is obtained in a similar way.

Proof of (32). We combine Lemma 3 with the inequalities

$$
\begin{aligned}
\tilde{\mathbf{E}} N_{1}^{+} & \leq \sum_{\{x, y, z\} \subset V} \sum_{w \in W} \frac{Y_{x} Y_{y} Y_{z} X_{w}^{3}}{(n m)^{3 / 2}}\left(\mathbb{I}_{\left\{Y_{x} Y_{y}>\varepsilon_{n} n\right.}+\mathbb{I}_{\left\{Y_{x} Y_{z}>\varepsilon_{n} n\right.}+\mathbb{I}_{\left\{Y_{y} Y_{z}>\varepsilon_{n} n\right.}\right) \\
\leq & 3 \beta^{-3 / 2} \hat{b}_{1} n^{-1} L_{n}^{\prime} S_{X}=o_{P}\left(S_{X}\right) \\
\tilde{\mathbf{E}} N_{2}^{+} & \leq \sum_{\Lambda} \sum_{w, \tau \in W: w \neq \tau} \frac{Y_{y} Y_{x}^{2} Y_{z} X_{w}^{2} X_{\tau}^{2}}{n^{2} m^{2}}\left(\mathbb{I}_{Y_{x} Y_{y}>\varepsilon_{n} n}+\mathbb{I}_{Y_{x} Y_{z}>\varepsilon_{n} n}\right) \\
& =\sum_{x \in V} \sum_{y, z \in V \backslash\{x\}: y \neq z} \sum_{w, \tau \in W: w \neq \tau} \frac{Y_{y} Y_{x}^{2} Y_{z} X_{w}^{2} X_{\tau}^{2}}{n^{2} m^{2}} \mathbb{I}_{Y_{x} Y_{y}>\varepsilon_{n} n} \\
& \leq \hat{a}_{2}^{2} \hat{b}_{1} L_{n}=o_{P}\left(S_{Y}\right) .
\end{aligned}
$$

In the last steps of (34) and (35) we have used (31).

Proof of (33). We combine Lemma 3 with the inequalities

$$
\begin{aligned}
\tilde{\mathbf{E}} N_{3}^{-} & \leq 3 ! \sum_{\{x, y, z\} \subset V} \sum_{\{w, \tau, \eta\} \subset W} \frac{Y_{x}^{2} Y_{y}^{2} Y_{z}^{2} X_{w}^{2} X_{\tau}^{2} X_{\eta}^{2}}{(n m)^{3}} \mathbb{I}_{Y_{x} Y_{y} \leq \varepsilon_{n} n} \\
& \leq \varepsilon_{n} \hat{a}_{2}^{3} \sum_{x, y, z \in V: x \neq y \neq z} \frac{Y_{x} Y_{y} Y_{z}^{2}}{n^{2}} \\
& \leq \varepsilon_{n} \hat{a}_{2}^{3} \hat{b}^{2} S_{Y}=o_{P}\left(S_{Y}\right), \\
\tilde{\mathbf{E}} N_{4}^{-} & \sum_{x, y, z \in V: x \neq y \neq z} \sum_{w, \tau, \eta \in W: w \neq \tau \neq \eta} \frac{Y_{x}^{2} Y_{y}^{3} Y_{z} X_{w}^{2} X_{\tau}^{2} X_{\eta}^{2}}{(n m)^{3}} \mathbb{I}_{Y_{x} Y_{y} \leq \varepsilon_{n} n} \\
\leq & \varepsilon_{n} \hat{a}_{2}^{3} \sum_{x, y, z \in V: x \neq y \neq z} \frac{Y_{x} Y_{y}^{2} Y_{z}}{n^{2}} \\
\leq & \varepsilon_{n} \hat{a}_{2}^{3} \hat{b}_{1}^{2} S_{Y}=o_{P}\left(S_{Y}\right), \sum_{x, y, z \in V: x \neq y \neq z} \sum_{w, \tau \in W: w \neq \tau} \frac{Y_{x}^{2} Y_{y}^{2} Y_{z} X_{w}^{3} X_{\tau}^{2}}{(n m)^{5 / 2}} \mathbb{I}_{Y_{x} Y_{y} \leq \varepsilon_{n} n} \\
& \leq \sum_{n} \hat{a}_{2} S_{X} \sum_{x, y, z \in V: x \neq y \neq z} \frac{Y_{x} Y_{y} Y_{z}}{\beta^{3 / 2} n^{3}} \\
& \leq \varepsilon_{n} \hat{a}_{2} \hat{b}_{1}^{3} \beta^{-3 / 2} S_{X}=o_{P}\left(S_{X}\right) .
\end{aligned}
$$

Now we are ready to prove 27.30$)$. 
Proof of (29). Given a light unlucky path $x \sim y \sim z$ of $G$, let $\mathcal{H}_{2}^{x, y, z} \subset \mathcal{H}_{2}$ denote the set of copies of $H_{2}$ defining this path. Fix an element $H_{2}^{*} \in \mathcal{H}_{2}^{x, y, z}$. All the other elements of $\mathcal{H}_{2}^{x, y, z}$ are called duplicates. We do this for each light unlucky path. We claim that the total number of duplicates is at most $N_{3}^{-}+N_{5}^{-}$. Indeed, given $H_{2}^{*} \in \mathcal{H}_{2}^{x, y, z}$ with bipartition denoted by $V^{\prime}=\{x, y, z\} \subset V$ and $W^{\prime}=\{w, \tau\} \subset W$, one potential duplicate is the distinct element of $\mathcal{H}_{2}^{x, y, z}$ with the same attribute set $W^{\prime}$. The union of both copies of $H_{2}$ defines the complete bipartite graph on $V^{\prime} \cup W^{\prime}$ and hence a copy of $H_{5}$ on $V^{\prime} \cup W^{\prime}$. The duplicates of this kind are counted by $N_{5}^{-}$. Remaining possible duplicates of $H_{2}^{*}$ have attribute sets different from $W^{\prime}$. We note that a duplicate $H_{2}^{\prime \prime}$ whose attribute set $W^{\prime \prime} \neq W^{\prime}$ defines a copy of $H_{3}$. Indeed, for $W^{\prime \prime} \cap W^{\prime}=\{w\}$ the union $H_{2}^{*} \cup H_{2}^{\prime \prime}$ is a copy of $H_{3}$. Furthermore, for $W^{\prime \prime} \cap W^{\prime}=\emptyset$, the union $H_{2}^{*} \cup H_{2}^{\prime \prime}$ with deleted vertex $w$ is a copy of $H_{3}$. Note that distinct duplicates $H_{2}^{\prime \prime}$ define distinct copies of $H_{3}$. Hence, their total number is at most $N_{3}^{-}$. Our claim is established. It implies that the number of light unlucky paths is at least $N_{2}^{-}-N_{3}^{-}-N_{5}^{-}$. Hence the total number of unlucky paths

$$
\Lambda_{U} \geq N_{2}^{-}-N_{3}^{-}-N_{5}^{-}=N_{2}-N_{2}^{+}-N_{3}^{-}-N_{5}^{-} \text {. }
$$

These inequalities in combination with (32), (33) and the simple inequality $\Lambda_{U} \leq N_{2}$ imply (29). Proof of (30). A light path $x \sim y \sim z$ receives both labels lucky and unlucky whenever $H$ has a light copy of $H_{1}$ with the vertex set $\{x, y, z\} \cup\{w\}$ and it has a light copy of $H_{2}$ with the vertex set $\{x, y, z\} \cup\left\{w^{\prime}, \tau\right\}$. Here $w$ and $w^{\prime} \neq \tau$ are arbitrary elements of $W$ not necessarily all distinct. The union of these two copies contains a light copy of $H_{5}$. Hence the number of light paths which are both lucky and unlucky is at most $N_{5}^{-}$. The number of heavy unlucky paths is at most $N_{2}^{+}$. Putting things together we obtain $\Lambda_{L U} \leq N_{5}^{-}+N_{2}^{+}$. Now (32), (33) imply (30). Proof of (28). Every heavy unlucky triangle contains at least two heavy unlucky paths. Hence the number of such triangles is at most $N_{2}^{+} / 2$. The number of light unlucky triangles is at most $N_{3}^{-}$. Hence $\Delta_{U} \leq N_{3}^{-}+N_{2}^{+} / 2=o_{P}\left(S_{Y}\right)$.

Proof of (27). Given a light lucky triangle $x \sim y \sim z \sim x$ of $G$, let $\mathcal{H}_{1}^{x, y, z} \subset \mathcal{H}_{1}$ denote the set of copies of $H_{1}$ defining this triangle. Fix an element $H_{1}^{*} \in \mathcal{H}_{1}^{x, y, z}$. It is the complete bipartite graph on the bipartition $\{x, y, z\} \cup\{w\}$ for some $w \in W$. All the other elements of $\mathcal{H}_{1}^{x, y, z}$ are called duplicates. We claim that the total number of duplicates is at most $N_{5}^{-}$. Indeed, for any duplicate $H_{1}^{\prime} \in \mathcal{H}_{1}^{x, y, z}$ with bipartition denoted by $\{x, y, z\} \cup\left\{w^{\prime}\right\}$, the union $H_{1}^{*} \cup H_{1}^{\prime}$ is the complete bipartite graph on $\{x, y, z\} \cup\left\{w, w^{\prime}\right\}$. We remove the edge $\left\{z, w^{\prime}\right\}$ and obtain a copy of $H_{5}$. We conclude that the number of light lucky triangles is at least $N_{1}^{-}-N_{5}^{-}$. Hence $\Delta_{L} \geq N_{1}^{-}-N_{5}^{-}=N_{1}-N_{1}^{+}-N_{5}^{-}$. These inequalities in combination with 32, 33 and the simple inequality $\Delta_{L} \leq N_{1}$ imply (27).

In the proof we use the fact that $n=o_{P}\left(S_{Y}\right)$ and $m=o_{P}\left(S_{X}\right)$.

Proof of Remark 1. For $\alpha<1$, random variables $S_{X}\left(c_{x} \Gamma(1-\alpha) m\right)^{-1 / \alpha}$ and $S_{Y}\left(c_{y} \Gamma(1-\alpha) n\right)^{-1 / \alpha}$ converge in distribution to independent and identically distributed $\alpha$ stable random variables, say $Z_{1}, Z_{2}$, having the Laplace transform $s \rightarrow \mathbf{E} e^{-s Z_{1}}=e^{-s^{\alpha}}$, see Theorem 2 of Section 6 of Chapter XIII of [7]. Here $\Gamma$ is Euler's Gamma function. Hence the statement (i).

For $\alpha=1$, there exist deterministic sequences $b_{m, x}=\left(c_{x}+o(1)\right) \ln m$ and $b_{n, y}=\left(c_{y}+o(1)\right) \ln n$ such that the random variables $m^{-1} S_{x}-b_{m, x}$ and $n^{-1} S_{Y}-b_{n, y}$ converge in distribution to independent asymmetric stable random variables with the characteristic exponent $\alpha=1$, see Theorem 3 of Section 5 of Chapter XVII of [7]. Hence the statement (ii). 


\section{Appendix}

In Appendix A we place auxiliary lemmas. Proofs are given in Appendix B. We remark that Lemmas 4 and 5, 6refer to the notation of the proofs of Theorems 2 and 3 respectively.

\subsection{Appendix A}

Lemma 1. Let $X_{1}, X_{2}, \ldots$ be a sequence of non-negative random variables converging in distribution to a random variable $X$. Assume that $\mathbf{E} X=\infty$. Then for some positive nonrandom sequence $\left\{\phi_{n}\right\}$ converging to $+\infty$ we have

$$
\mathbf{P}\left(X_{n, 1}+\cdots+X_{n, n}>\phi_{n} n\right)=1-o(1) .
$$

Here $X_{n, 1}, \ldots, X_{n, n}$ are iid copies of $X_{n}$.

Lemma 2. Let $\left\{Z_{n}\right\}$ and $\eta=\left\{\eta_{n}\right\}$ be sequences of random variables defined on the same probability space. Let $\mathbf{E}_{\eta}$ denote the conditional expectation given $\eta$. Assume that $\mathbf{E}_{\eta} Z_{n}=0$ implies $Z_{n}=0$. Then

(i) $\mathbf{E}_{\eta}\left(Z_{n}-\mathbf{E}_{\eta} Z_{n}\right)^{2}=o_{P}\left(\left(\mathbf{E}_{\eta} Z_{n}\right)^{2}\right)$ implies $Z_{n}=\left(1+o_{P}(1)\right) \mathbf{E}_{\eta} Z_{n}$;

(ii) $Z_{n}=O_{P}\left(\mathbf{E}_{\eta} Z_{n}\right)$.

Lemma 3. Let $t>0$. Let $Z$ be a non-negative random variable with $\mathbf{E} Z<\infty$.

(i) There exists a positive increasing function $\psi(\cdot)$ such $\psi(t) \uparrow+\infty$ as $t \uparrow+\infty$ and $\mathbf{E} Z \psi(Z)<\infty$. Furthermore, there exists a positive decreasing function $\varepsilon(\cdot)$ such that $\varepsilon(s) \downarrow 0$ as $s \uparrow+\infty$ and $\mathbf{P}(Z>s \varepsilon(s))=o\left(s^{-1}\right)$ for $s \rightarrow+\infty$.

(ii) Let $Z_{1}, Z_{2}, \ldots$ be iid copies of $Z$. Let $n \rightarrow+\infty$. Then $Z_{1}^{1+t}+\cdots+Z_{n}^{1+t}=o_{P}\left(n^{1+t}\right)$. Furthermore, for $\varepsilon(\cdot)$ of statement (i), we have $\mathbf{P}\left(\max _{1 \leq i \leq n} Z_{i}>n \varepsilon(n)\right)=o(1)$.

We remark that the functions $\psi(\cdot), \varepsilon(\cdot)$ depend on the probability distribution of $Z$.

Lemma 4. Let $X_{1}, X_{2}, \ldots$ be a sequence of non-negative random variables converging in distribution to a random variable $X$. Assume that $\mathbf{E} X^{3}=\infty$ and $0<\mathbf{E} X^{2}<\infty$. Assume that $\mathbf{E} X_{n}^{2}<\infty$, for each $n=1,2, \ldots$, and $\lim _{n} \mathbf{E} X_{n}^{2}=\mathbf{E} X^{2}$. Let $\left\{m_{n}, n \geq 1\right\}$ be an integer sequence and, for every $n$, let $X_{n, 1}, \ldots, X_{n, m_{n}}$ be iid copies of $X_{n}$. Let $n \rightarrow+\infty$. Assume that $m_{n} \uparrow+\infty$. Denote $S_{X, n}=\sum_{j \in\left[m_{n}\right]} X_{n, j}^{3}$. We have

$$
\begin{aligned}
m_{n}^{-3} \sum_{\{j, k\} \subset\left[m_{n}\right]} X_{n, j}^{3} X_{n, k}^{3} & =o_{P}\left(S_{X, n}\right), \\
m_{n}^{-3} \sum_{\{j, k, r\} \subset\left[m_{n}\right]} X_{n, j}^{2} X_{n, k}^{2} X_{n, r}^{2} & =o_{P}\left(S_{X, n}\right), \\
m_{n}^{-1} \sum_{\{j, k\} \subset\left[m_{n}\right]} X_{n, j}^{2} X_{n, k}^{2} & =o_{P}\left(S_{X, n}\right) .
\end{aligned}
$$

Lemma 5. Assume that $\mathbf{E} X_{1}^{2}<\infty$ and $\mathbf{E} Y_{1}<\infty$.

(i) For $\mathbf{E} X_{1}^{3}=\infty$ we have $\tilde{\mathbf{E}}\left(N_{1}-\tilde{\mathbf{E}} N_{1}\right)^{2}=o_{P}\left(S_{X}^{2}\right)$.

(ii) We have $\tilde{\mathbf{E}} N_{1}=6^{-1} \beta^{-3 / 2} b_{1}^{3} S_{X}+o_{P}\left(S_{X}\right)$.

Lemma 6. Assume that $\mathbf{E} X_{1}^{2}<\infty, \mathbf{E} Y_{1}<\infty$.

(i) For $\mathbf{E} Y_{1}^{2}=\infty$ we have $\tilde{\mathbf{E}}\left(N_{2}-\tilde{\mathbf{E}} N_{2}\right)^{2}=o_{P}\left(S_{Y}^{2}\right)$.

(ii) We have $\tilde{\mathbf{E}} N_{2}=2^{-1} a_{2}^{2} b_{1}^{2} S_{Y}+o_{P}\left(S_{Y}\right)$. 


\subsection{Appendix B}

Proof of Lemma 1. We need some notation. Given random variable $Z$ and sequence $Z_{1}, \ldots, Z_{N}$ of iid copies of $Z$, we denote $S_{N}(Z)=Z_{1}+\cdots+Z_{N}$. For a constant $A>0$ we denote the truncated random variable $Z[A]=Z \mathbb{I}_{\{Z \leq A\}}$ and $S_{N}(Z[A])$ denotes the sum of truncated iid copies of $Z$.

Let us prove (36). Choose a sequence $\left\{A_{n}\right\}$ of positive constants converging to $+\infty$ (slowly enough) such that

$$
\operatorname{Var}\left(X\left[A_{n}\right]\right)=o(n) \quad \text { and } \quad \mathbf{E} X_{n}^{i}\left[A_{n}\right]-\mathbf{E} X^{i}\left[A_{n}\right]=o(1), \quad i=1,2 .
$$

In particular, we have $\mathbf{E} X_{n}\left[A_{n}\right] \rightarrow+\infty$ and $\operatorname{Var} X_{n}\left[A_{n}\right]=o(n)$ as $n \rightarrow+\infty$. Now Chebyshev's inequality implies

$$
\mathbf{P}\left(S_{n}\left(X_{n}\left[A_{n}\right]\right)<(n / 2) \mathbf{E} X_{n}\left[A_{n}\right]\right) \leq 4 n^{-1}\left(\mathbf{E} X_{n}\left[A_{n}\right]\right)^{-2} \operatorname{Var} X_{n}\left[A_{n}\right]=o(1) .
$$

Hence, for $S_{n}\left(X_{n}\right) \geq S_{n}\left(X_{n}\left[A_{n}\right]\right)$ and $\phi_{n}=0.5 \mathbf{E} X_{n}\left[A_{n}\right]$ we obtain $\mathbf{P}\left(S_{n}\left(X_{n}\right)<\phi_{n} n\right)=o(1)$.

Proof of Lemma 2. Let $\mathbf{P}_{\eta}$ denote the conditional probability given $\eta$ and let $z_{n}$ denote $\mathbf{E}_{\eta} Z_{n}$. We obtain (i) by Chebyshev's inequality: $\forall \varepsilon>0$

$$
\begin{aligned}
\mathbf{P}\left(\left|Z_{n}-z_{n}\right|>\varepsilon z_{n}\right) & =\mathbf{E} \min \left\{1, \mathbf{P}_{\eta}\left(\left|Z_{n}-z_{n}\right|>\varepsilon z_{n}\right)\right\} \mathbb{I}_{\left\{z_{n} \neq 0\right\}} \\
& \leq \mathbf{E} \min \left\{1,\left(\varepsilon z_{n}\right)^{-2} \mathbf{E}_{\eta}\left(Z_{n}-z_{n}\right)^{2}\right\} \mathbb{I}_{\left\{z_{n} \neq 0\right\}} \\
& =o(1) .
\end{aligned}
$$

In the last step we used the fact that $\mathbf{P}\left(\mathbf{E}_{\eta}\left(Z_{n}-z_{n}\right)^{2}>\delta z_{n}^{2}\right)=o(1)$ for any $\delta>0$.

We obtain (ii) by Markov's inequality: $\forall \varepsilon>0$

$$
\mathbf{P}\left(Z_{n}>\varepsilon^{-1} z_{n}\right)=\mathbf{E}\left(\mathbf{P}_{\eta}\left(Z_{n}>\varepsilon^{-1} z_{n}\right) \mathbb{I}_{\left\{z_{n} \neq 0\right\}}\right) \leq \mathbf{E}\left(\varepsilon z_{n}^{-1} \mathbf{E}_{\eta} Z_{n}\right) \mathbb{I}_{\left\{z_{n} \neq 0\right\}} \leq \varepsilon .
$$

Proof of Lemma 3. The poof is elementary. We present it for reader's convenience.

Proof of (i). $\mathbf{E} Z<\infty$ implies that the function $\phi(t)=\mathbf{E} Z \mathbb{I}_{\{Z>t\}}$ is non-increasing and $\phi(t) \rightarrow 0$ as $t \rightarrow+\infty$. Choose an increasing positive sequence $\left\{s_{k}\right\}_{k \geq 1}$ such that $s_{k} \uparrow+\infty$ and $\phi\left(s_{k}\right) \leq 2^{-k}$ and $s_{1} \geq 1$. Put $s_{0}=0$. Consider the non-decreasing function $\psi:[0,+\infty) \rightarrow[0,+\infty)$ attaining value $k$ on the interval $\left[s_{k-1}, s_{k}\right]$, for $k=1,2 \ldots$ Clearly, $\psi(t) \rightarrow+\infty$ as $t \uparrow+\infty$ and we have $\mathbf{E} Z \psi(Z)<\infty$. Furthermore, we can easily modify $\psi(\cdot)$ in order to obtain a strictly increasing function satisfying the requirements of statement (i). Now we choose $\varepsilon(\cdot)$ decaying slowly enough $(\varepsilon(s) \downarrow 0$ as $s \uparrow+\infty)$ so that $s \varepsilon(s) \rightarrow+\infty$ and $\varepsilon(s) \psi(s \varepsilon(s)) \rightarrow+\infty$ as $s \rightarrow+\infty$. Finally, Markov's inequality implies

$$
\mathbf{P}(Z>s \varepsilon(s)) \leq(s \varepsilon(s) \psi(s \varepsilon(s)))^{-1} \mathbf{E} Z \psi(Z) \mathbb{I}_{Z \psi(Z)>s \varepsilon(s)}=o\left(s^{-1}\right) .
$$

Proof of (ii). We write $Z_{1}^{1+t}+\cdots+Z_{n}^{1+t} \leq A B$, where $A:=Z_{1}+\cdots+Z_{n}$ and $B:=\max _{i \in[n]} Z_{i}^{t}$, and invoke the bounds $A=O_{P}(n)$ and $B=o_{P}\left(n^{t}\right)$. The first bound follows by the law of large numbers. The second one follows by Markov's inequality and the union bound: $\forall \delta>0$ we have

$$
\mathbf{P}\left(B>\delta^{t} n^{t}\right)=\mathbf{P}\left(\max _{i \in[n]} Z_{i}>\delta n\right) \leq n \mathbf{P}\left(Z_{1}>\delta n\right) \leq \delta^{-1} \mathbf{E} Z_{1} \mathbb{I}_{Z_{1}>\delta n}=o(1) .
$$

Similarly, from (i) we obtain $\mathbf{P}\left(\max _{i \in[n]} Z_{i}>n \varepsilon(n)\right) \leq n \mathbf{P}\left(Z_{1}>n \varepsilon(n)\right)=o(1)$. 
Proof of Lemma 4. Proof of 37. The event $\mathcal{A}_{n}=\left\{\max _{j \in\left[m_{n}\right]} X_{j} \geq m_{n}^{4 / 7}\right\}$ has probability

$$
\mathbf{P}\left(\mathcal{A}_{n}\right) \leq m_{n} \mathbf{P}\left(X_{n, j} \geq m_{n}^{4 / 7}\right) \leq m_{n}^{-1 / 7} \mathbf{E} X_{n, j}^{2}=o(1) .
$$

On the complement event, the left side of (37) is less than

$$
m_{n}^{-3} \sum_{\{j, k\} \subset\left[m_{n}\right]} m_{n}^{12 / 7} X_{n, k}^{3} \leq m_{n}^{-2 / 7} S_{X, n} .
$$

Proof of 38 . Denote $S_{* n}=m_{n}^{-1} \sum_{i \in\left[m_{n}\right]} X_{n, j}^{2}$. The relation $\mathbf{E} S_{* n}=\mathbf{E} X_{n}^{2} \rightarrow \mathbf{E} X^{2}$ implies $S_{* n}=O_{P}(1)$. The left side of 38 is less than $S_{* n}^{3}=O_{P}(1)$. The right side is superlinear in $m$, by Lemma 1.

Proof of $(39)$. The left side of 39 is less than $m_{n} S_{* n}^{2}=O_{P}\left(m_{n}\right)$. The right side is superlinear in $m$, by Lemma 1 .

Proof of Lemma 5. It is convenient to write $N_{1}$ in the form

$$
N_{1}=\sum_{w \in W} U_{w}, \quad U_{w}:=\sum_{\{x, y, z\} \subset V} \mathbb{I}_{x w} \mathbb{I}_{y w} \mathbb{I}_{z w} .
$$

Proof of (i). Given $\mathbb{X}$ and $\mathbb{Y}$, the random variables $U_{w}, w \in W$ are conditionally independent. Hence

$$
\tilde{\mathbf{E}}\left(N_{1}-\tilde{\mathbf{E}} N_{1}\right)^{2}=\sum_{w \in W} \tilde{\mathbf{E}} \tilde{U}_{w}^{2}, \quad \text { where } \quad \tilde{U}_{w}:=U_{w}-\tilde{\mathbf{E}} U_{w}
$$

We bound every expectation $\tilde{\mathbf{E}} \tilde{U}_{w}^{2}$ using conditional Hoeffding's decomposition $\tilde{U}_{w}=L_{w}+Q_{w}+$ $K_{w}$, where the components

$$
\begin{aligned}
L_{w} & =\sum_{x \in V} \tilde{\mathbf{E}}\left(\tilde{U}_{w} \mid \mathbb{I}_{x w}\right), \quad Q_{w}=\sum_{\{x, y\} \subset V} \tilde{\mathbf{E}}\left(\tilde{U}_{w}-L_{w} \mid \mathbb{I}_{x w}, \mathbb{I}_{y w}\right), \\
K_{w} & =\sum_{\{x, y, z\} \subset V} \tilde{\mathbf{E}}\left(\tilde{U}_{w}-L_{w}-Q_{w} \mid \mathbb{I}_{x w}, \mathbb{I}_{y w}, \mathbb{I}_{z w}\right),
\end{aligned}
$$

called the linear, quadratic and cubic part of the decomposition, are conditionally uncorrelated. We have in particular that

$$
\tilde{\mathbf{E}} \tilde{U}_{w}^{2}=\tilde{\mathbf{E}} L_{w}^{2}+\tilde{\mathbf{E}} Q_{w}^{2}+\tilde{\mathbf{E}} K_{w}^{2} .
$$

Moreover the summands of all three sums of 42 are conditionally uncorrelated (given $\mathbb{X}, \mathbb{Y}$. Now (i) follows from (41) and the bounds shown below

$$
\sum_{w \in W} \tilde{\mathbf{E}} L_{w}^{2}=o_{P}\left(S_{X}^{2}\right), \quad \sum_{w \in W} \tilde{\mathbf{E}} Q_{w}^{2}=o_{P}\left(S_{X}^{2}\right), \quad \sum_{w \in W} \tilde{\mathbf{E}} K_{w}^{2}=o_{P}\left(S_{X}^{2}\right) .
$$

Let us prove (43). Denote, for $x, y, z \in V$ and $w \in W$,

$$
s_{x \mid w}=\sum_{\{y, z\} \subset V \backslash\{x\}} p_{y w} p_{z w}, \quad s_{x y \mid w}=\sum_{z \in V \backslash\{x, y\}} p_{z w} .
$$

A straightforward calculation shows that

$$
\begin{aligned}
& \tilde{\mathbf{E}}\left(\tilde{U}_{w} \mid \mathbb{I}_{x w}\right)=\left(\mathbb{I}_{x w}-p_{x w}\right) s_{x \mid w}=: l_{w}(x), \\
& \tilde{\mathbf{E}}\left(\tilde{U}_{w}-L_{w} \mid \mathbb{I}_{x w}, \mathbb{I}_{y w}\right)=\left(\mathbb{I}_{x w}-p_{x w}\right)\left(\mathbb{I}_{y w}-p_{y w}\right) s_{x y \mid w}=: q_{w}(x, y), \\
& \tilde{\mathbf{E}}\left(\tilde{U}_{w}-L_{w}-Q_{w} \mid \mathbb{I}_{x w}, \mathbb{I}_{y w}, \mathbb{I}_{z w}\right)=\left(\mathbb{I}_{x w}-p_{x w}\right)\left(\mathbb{I}_{y w}-p_{y w}\right)\left(\mathbb{I}_{z w}-p_{z w}\right)=: k_{w}(x, y, z) .
\end{aligned}
$$


Invoking the simple inequalities $s_{x \mid w} \leq X_{w}^{2} \beta_{n}^{-1} \hat{b}_{1}^{2}$ and $s_{x y \mid w} \leq X_{w} \beta_{n}^{-1 / 2} \hat{b}_{1}$ we obtain

$$
\begin{aligned}
\tilde{\mathbf{E}} l_{w}^{2}(x) & =\tilde{\mathbf{E}}\left(\left(\mathbb{I}_{x w}-p_{x w}\right) s_{x \mid w}\right)^{2} \leq p_{x w} s_{x \mid w}^{2} \leq X_{w}^{5} \beta_{n}^{-5 / 2} Y_{x} n^{-1} \hat{b}_{1}^{4}, \\
\tilde{\mathbf{E}} q_{w}^{2}(x, y) & =\tilde{\mathbf{E}}\left(\left(\mathbb{I}_{x w}-p_{x w}\right)\left(\mathbb{I}_{y w}-p_{y w}\right) s_{x y \mid w}\right)^{2} \leq p_{x w} p_{y w} s_{x y \mid w}^{2} \leq X_{w}^{4} \beta_{n}^{-2} n^{-2} Y_{x} Y_{y} \hat{b}_{1}^{2}, \\
\tilde{\mathbf{E}} k_{w}^{2}(x, y, z) & \leq p_{x w} p_{y w} p_{z w} \leq X_{w}^{3} \beta_{n}^{-3 / 2} n^{-3} Y_{x} Y_{y} Y_{z} .
\end{aligned}
$$

We note that for $x, y, z \in V$ the random variables $l_{w}(x), q_{w}(x, y)$ and $k_{w}(x, y, z)$ are uncorrelated. Hence

$$
\tilde{\mathbf{E}} L_{w}^{2}=\sum_{x \in V} \tilde{\mathbf{E}} l_{w}^{2}(x), \quad \tilde{\mathbf{E}} Q_{w}^{2}=\sum_{\{x, y\} \subset V} \tilde{\mathbf{E}} q_{w}^{2}(x, y), \quad \tilde{\mathbf{E}} K_{w}^{2}=\sum_{\{x, y, z\} \subset V} \tilde{\mathbf{E}} k_{w}^{2}(x, y, z)
$$

Now from 44 we obtain the bounds

$$
\tilde{\mathbf{E}} L_{w}^{2}=O_{P}\left(\sum_{w \in W} X_{w}^{5}\right), \quad \tilde{\mathbf{E}} Q_{w}^{2}=O_{P}\left(\sum_{w \in W} X_{w}^{4}\right), \quad \tilde{\mathbf{E}} K_{w}^{2}=O_{P}\left(\sum_{w \in W} X_{w}^{3}\right) .
$$

Next, we apply Hölder's inequality. For $r=3,4,5$, we have

$$
\left(\sum_{w \in W} 1 \cdot X_{w}^{r}\right)^{6 / r} \leq\left(\sum_{w \in W} 1\right)^{(6-r) / r} \sum_{w \in W} X_{w}^{6} \leq m^{(6-r) / r} S_{X}^{2} .
$$

Finally, from the bound $m=o_{P}\left(S_{X}\right)$, which holds for $\mathbf{E} X_{1}^{3}=\infty$, see Lemma 1, we obtain

$$
\sum_{w \in W} X_{w}^{r} \leq m^{(6-r) / 6} S_{X}^{r / 3}=o_{P}\left(S_{X}^{(6-r) / 6}\right) S_{X}^{r / 3}=o_{P}\left(S_{X}^{2}\right) .
$$

Proof of (ii). Denote $H_{w}=\sum_{x \in V} \lambda_{x w}$ and $R_{w}=H_{w}^{3}-6 \tilde{\mathbf{E}} U_{w}$. We have

$$
\tilde{\mathbf{E}} N=\sum_{w \in W} \tilde{\mathbf{E}} U_{w}=6^{-1} \sum_{w \in W} H_{w}^{3}-6^{-1} \sum_{w \in W} R_{w} .
$$

A straightforward calculation shows that

$$
\sum_{w \in W} H_{w}^{3}=\beta_{n}^{-3 / 2} \hat{b}_{1}^{3} S_{X}=\left(1+o_{P}(1)\right) \beta^{-3 / 2} b_{1}^{3} S_{X}
$$

Hence, it remains to prove that $\sum_{w \in W} R_{w}=o_{P}\left(S_{X}\right)$. To show this bound we write $R_{w}=$ $R_{1, w}+R_{2, w}$, where

$$
R_{1, w}=H_{w}^{3}-Z_{w}^{3}, \quad R_{2, w}=Z_{w}^{3}-6 \tilde{\mathbf{E}} U_{w}, \quad \text { and } \quad Z_{w}=\sum_{x \in V} p_{x w}
$$

and establish the bounds

$$
\sum_{w \in W} R_{1, w}=o_{P}\left(S_{X}\right) \quad \text { and } \quad \sum_{w \in W} R_{2, w}=o_{P}\left(S_{X}\right) .
$$

We first prove the second bound of (45). We have

$$
0 \leq R_{2, w}=\sum_{x \in V} p_{x w}^{3}+3 \sum_{x \in V} \sum_{y \in V \backslash\{x\}} p_{x w}^{2} p_{y w} \leq \beta_{n}^{-3 / 2} X_{w}^{3}\left(n^{-2} \hat{b}_{3}+3 n^{-1} \hat{b}_{1} \hat{b}_{2}\right) .
$$


In the last step we used $p_{x w} \leq \lambda_{x w}$. Next, invoking the bounds $n^{-2} \hat{b}_{1}^{3}, n^{-1} \hat{b}_{2}=o_{P}(1)$, which hold for $\mathbf{E} Y_{1}<\infty$, by Lemma (3), we obtain

$$
0 \leq \sum_{w \in W} R_{2, w} \leq \beta_{n}^{-3 / 2} S_{X}\left(n^{-2} \hat{b}_{3}+3 n^{-1} \hat{b}_{1} \hat{b}_{2}\right)=o_{P}\left(S_{X}\right)
$$

Let us prove the first bound of 45 . We note that $\mathbf{E} X_{1}^{2}<\infty, \mathbf{E} Y_{1}<\infty$ imply that

$$
\mathbf{E} X_{1}^{2} \mathbb{I}_{\left\{X_{1}>\sqrt{m}\right\}} \rightarrow 0, \quad \mathbf{E} Y_{1} \mathbb{I}_{\left\{Y_{1}>\sqrt{n}\right\}} \rightarrow 0 .
$$

We select a sequence $\delta_{n} \downarrow 0$ such that $\mathbf{E} Y_{1} \mathbb{I}_{\left\{Y_{1}>\sqrt{n}\right\}}=o\left(\delta_{n}\right)$ and introduce events

$$
\mathcal{A}=\left\{\max _{i \in[m]} X_{i} \leq \sqrt{m}\right\}, \quad \mathcal{B}=\left\{n^{-1} \sum_{j \in[n]} Y_{j} \mathbb{I}_{\left\{Y_{j}>\sqrt{n}\right\}} \leq \delta_{n}\right\} .
$$

We claim that $\mathbf{P}(\mathcal{A}), \mathbf{P}(\mathcal{B})=1-o(1)$. Indeed, by Markov's inequality and 47)

$$
\begin{aligned}
& 1-\mathbf{P}(\mathcal{A}) \leq \sum_{i \in[m]} \mathbf{P}\left(X_{i}>\sqrt{m}\right) \leq \mathbf{E} X_{1}^{2} \mathbb{I}_{\left\{X_{1}>\sqrt{m}\right\}} \rightarrow 0 \\
& 1-\mathbf{P}(\mathcal{B}) \leq\left(n \delta_{n}\right)^{-1} \sum_{j \in[n]} \mathbf{E} Y_{j} \mathbb{I}_{\left\{Y_{j}>\sqrt{n}\right\}}=\delta_{n}^{-1} \mathbf{E} Y_{1} \mathbb{I}_{\left\{Y_{1}>\sqrt{n}\right\}} \rightarrow 0 .
\end{aligned}
$$

Assuming that events $\mathcal{A}$ and $\mathcal{B}$ hold we estimate the difference

$$
H_{w}-Z_{w}=\sum_{x \in V}\left(\lambda_{x w}-1\right) \mathbb{I}_{\left\{\lambda_{x w}>1\right\}} \leq \sum_{x \in V} \lambda_{x w} \mathbb{I}_{\left\{Y_{x}>\sqrt{n}\right\}} \leq X_{w} \beta_{n}^{-1 / 2} \delta_{n} .
$$

Here we used the inequality $\mathbb{I}_{\left\{\lambda_{x w}>1\right\}} \leq \mathbb{I}_{\left\{Y_{x}>\sqrt{n}\right\}}$, which holds for $X_{w} \leq \sqrt{m}$. Invoking (48) in the inequalities

$$
0 \leq R_{1, w}=\left(H_{w}-Z_{w}\right)\left(Z_{w}^{2}+Z_{w} H_{w}+H_{w}^{2}\right) \leq 3\left(H_{w}-Z_{w}\right) H_{w}^{2},
$$

and using the identity $H_{w}^{2}=X_{w}^{2} \beta_{n}^{-1} \hat{b}_{1}^{2}$, we obtain

$$
\sum_{w \in W} R_{1, w} \leq S_{X} \beta_{n}^{-3 / 2} \hat{b}_{1}^{2} \delta_{n}
$$

For the latter inequality holds with probability $1-o(1)$ and $\delta_{n}=o(1)$, we conclude that $\sum_{w \in W} R_{1, w}=o_{P}\left(S_{X}\right)$.

Proof of Lemma 6. Proof of (i). In the proof we make use of Hoeffding's decomposition. Let $\mathbb{I}_{j}$, $j \in[4]$ be independent Bernoulli random variables with positive success probabilities $p_{j}, j \in[4]$. Hoeffding's decomposition represents the random variable $T=\mathbb{I}_{1} \mathbb{I}_{2} \mathbb{I}_{3} \mathbb{I}_{4}-p_{1} p_{2} p_{3} p_{4}$ by the sum of uncorrelated $U$ statistics of increasing order

$$
T=U_{1}+U_{2}+U_{3}+U_{4}, \quad U_{1}=\sum_{j \in[4]} T_{j}, \quad U_{2}=\sum_{\{i, j\} \subset[4]} T_{i j}, \quad U_{3}=\sum_{\{i, j, k\} \subset[4]} T_{i j k} .
$$

The first, second, and third order terms $T_{i}, T_{i j}$, and $T_{i j k}$ are defined iteratively as follows

$$
T_{i}=\mathbf{E}\left(T \mid \mathbb{I}_{i}\right), \quad T_{i j}=\mathbf{E}\left(T-U_{1} \mid \mathbb{I}_{i}, \mathbb{I}_{j}\right), \quad T_{i j k}=\mathbf{E}\left(T-U_{1}-U_{2} \mid \mathbb{I}_{i}, \mathbb{I}_{j}, \mathbb{I}_{k}\right) .
$$


Denoting $p=p_{1} p_{2} p_{3} p_{4}$ and $p_{i}^{*}=p / p_{i}, p_{i j}^{*}=p /\left(p_{i} p_{j}\right), p_{i j k}^{*}=p /\left(p_{i} p_{j} p_{k}\right)$ we have

$$
\begin{aligned}
& T_{i}=\left(\mathbb{I}_{i}-p_{i}\right) p_{i}^{*}, \quad T_{i j}=\left(\mathbb{I}_{i} \mathbb{I}_{j}-p_{i} p_{j}\right) p_{i j}^{*}-T_{i}-T_{j}, \\
& T_{i j k}=\left(\mathbb{I}_{i} \mathbb{I}_{j} \mathbb{I}_{k}-p_{i} p_{j} p_{k}\right) p_{i j k}^{*}-T_{i j}-T_{i k}-T_{j k}-T_{i}-T_{j}-T_{k} .
\end{aligned}
$$

The fourth order term $U_{4}=T_{1234}:=T-U_{1}-U_{2}-U_{3}$. We note that various terms of Hoeffding's decomposition are mutually uncorrelated.

Let us prove the lemma. Denote $\Lambda_{*}=N_{2}-\tilde{\mathbf{E}} N_{2}$ and $T_{(w, \tau)}^{y x z}=\mathbb{I}_{y w} \mathbb{I}_{x w} \mathbb{I}_{x \tau} \mathbb{I}_{z \tau}-\tilde{\mathbf{E}} \mathbb{I}_{y w} \mathbb{I}_{x w} \mathbb{I}_{x \tau} \mathbb{I}_{z \tau}$. We have

$$
\Lambda_{*}=\sum_{x \in V} \sum_{\{y, z\} \subset V \backslash\{x\}} \sum_{w \in W} \sum_{\tau \in W \backslash\{w\}} T_{(w, \tau)}^{y x z} .
$$

We decompose every $T_{(w, \tau)}^{y x z}$ using $(49)$ and invoke these decompositions in 51 . We then group the first order terms, the second order terms, etc. and obtain Hoeffding's decomposition of $\Lambda_{*}$,

$$
\Lambda_{*}=U_{1}^{*}+U_{2}^{*}+U_{3}^{*}+U_{4}^{*} .
$$

We specify the linear part $U_{1}^{*}$ (the sum of the first order terms), quadratic part $U_{2}^{*}$ (the sum of the second order terms), etc. in (52) below. For this purpose we introduce some more notation. Consider the complete bipartite graph $\mathcal{K}_{V, W}$ with the bipartition $V \cup W$. Let $\mathcal{E}=\{(y, w): y \in$ $V, w \in W\}$ denote the set of edges of $\mathcal{K}_{V, W}$. Let $\mathcal{E}^{*}$ denote the set of paths of length 4 which start from $V$. After we remove an edge of such a path we obtain a triple of edges, which we call trunk. The set of trunks is denoted $\mathcal{E}^{* *}$. For any edge $a=(y w) \in \mathcal{E}$ we denote $\mathbb{I}_{a}=\mathbb{I}_{y w}$ the indicator of the event that vertex $y$ is linked to the attribute $w$ in the random bipartite graph $H$. We also denote $p_{a}=\tilde{\mathbf{E}} \mathbb{I}_{a}$. Furthermore for distinct edges $a, b, c, d \in \mathcal{E}$ we denote

$$
\begin{aligned}
t_{a} & =\mathbb{I}_{a}-p_{a}, \quad t_{a b}=\left(\mathbb{I}_{a} \mathbb{I}_{b}-p_{a} p_{b}\right)-\left(\mathbb{I}_{a}-p_{a}\right) p_{b}-\left(\mathbb{I}_{b}-p_{b}\right) p_{a} \\
t_{a b c} & =\left(\mathbb{I}_{a} \mathbb{I}_{b} \mathbb{I}_{c}-p_{a} p_{b} p_{c}\right)-t_{a b} p_{c}-t_{a c} p_{b}-t_{b c} p_{a}-t_{a} p_{b} p_{c}-t_{b} p_{a} p_{c}-t_{c} p_{a} p_{b} .
\end{aligned}
$$

Finally, $t_{a b c d}$ is defined as $T_{1234}$ above, but for $T=\mathbb{I}_{a} \mathbb{I}_{b} \mathbb{I}_{c} \mathbb{I}_{d}-p_{a} p_{b} p_{c} p_{d}$.

A calculation shows that

$$
\begin{aligned}
U_{1}^{*} & =\sum_{a \in \mathcal{E}} t_{a} Q_{a}, & U_{2}^{*} & =\sum_{\{a, b\} \subset \mathcal{E}} t_{a b} Q_{a b}, \\
U_{3}^{*} & =\sum_{\{a, b, c\} \in \mathcal{E}^{* *}} t_{a b c} Q_{a b c}, & U_{4}^{*} & =\sum_{\{a, b, c, d\} \in \mathcal{E}^{*}} t_{a b c d},
\end{aligned}
$$

where coefficients $Q_{a}, Q_{a b}$ and $Q_{a b c}$ are given below. For any $a=(y, w)$ we have

$$
\begin{aligned}
& Q_{a}=Q_{a 1}+Q_{a 2}, \\
& Q_{a 1}=\sum_{x \in V \backslash\{y\}} p_{x w} \sum_{\tau \in W \backslash\{w\}} p_{x \tau} \sum_{z \in V \backslash\{x, y\}} p_{z \tau}, \quad Q_{a 2}=\sum_{x \in V \backslash\{y\}} p_{x w} \sum_{\tau \in W \backslash\{w\}} p_{y \tau} \sum_{z \in V \backslash\{x, y\}} p_{z \tau} .
\end{aligned}
$$

We note that sums $Q_{a 1}$ and $Q_{a 2}$ represent 4-paths, where $y$ has degree 1 and degree 2 respectively (e.g., paths $y \sim w \sim x \sim \tau \sim z$ and $x \sim w \sim y \sim \tau \sim z$ ). Furthermore, for a non incident pair $a=(y, w)$ and $c=(x, \tau)$ we have

$$
\begin{aligned}
& Q_{a c}=Q_{a c 1}+Q_{a c 2}+Q_{a c 3} \\
& Q_{a c 1}=p_{y \tau} \sum_{z \in V \backslash\{y, x\}} p_{z \omega}, \quad Q_{a c 2}=p_{x w} \sum_{z \in V \backslash\{y, x\}} p_{z \tau}, \quad Q_{a c 3}=\sum_{z \in V \backslash\{y, x\}} p_{z w} p_{z \tau},
\end{aligned}
$$


The sum $Q_{a c 1}\left(Q_{a c 2}\right)$ represents 4-paths, where $y(x)$ has degree 2 (e.g., paths $x \sim \tau \sim y \sim w \sim z$ and $y \sim w \sim x \sim \tau \sim z$ ). The sum $Q_{a c 3}$ represents 4-paths, where $y$ and $x$ has degree 1 (e.g., paths $y \sim w \sim z \sim \tau \sim x)$. Similarly, for incident pairs $a=(y, w), b=(x, w)$ and $b=(x, w)$, $c=(x, \tau)$ we have

$$
Q_{a b}=\sum_{z \in V \backslash\{x, y\}} \sum_{\tau \in W \backslash\{w\}}\left(p_{x \tau} p_{z \tau}+p_{y \tau} p_{z \tau}\right) \quad Q_{b c}=\sum_{\{y, z\} \subset V \backslash\{x\}}\left(p_{y w} p_{z \tau}+p_{z w} p_{y \tau}\right) .
$$

Finally, for a trunk $\{a, b, c\}$ which makes up a 3-path, say, $a=(y w), b=(x w), c=(x \tau)$, we have $Q_{a, b, c}=\sum_{z \in V \backslash\{x, y\}} p_{z \tau}$. For a trunk $\{a, b, d\}$ which is not a path (a union of 2-path and an edge), say, $a=(y w), b=(x w)$ and $d=(z \tau)$, we have $Q_{a, b, d}=p_{x \tau}$.

Now we estimate $\tilde{\mathbf{E}} \Lambda_{*}^{2}$. From the fundamental property of Hoeffding's decomposition that various terms are uncorrelated we obtain that

$$
\tilde{\mathbf{E}} \Lambda_{*}^{2}=\sum_{a \in \mathcal{E}} Q_{a}^{2} \tilde{E} t_{a}^{2}+\sum_{\{a, b\} \subset \mathcal{E}} Q_{a b}^{2} \tilde{E} t_{a b}^{2}+\sum_{\{a, b, c\} \in \mathcal{E}^{* *}} Q_{a b c}^{2} \tilde{E} t_{a b c}^{2}+\sum_{\{a, b, c, d\} \subset \mathcal{E}^{*}} \tilde{E} t_{a b c d}^{2}
$$

It remains to show that the sums in the right, which we denote by $Z_{1}, Z_{2}, Z_{3}, Z_{4}$, are of order $o_{P}\left(S_{Y}^{2}\right)$. For this purpose we combine the expressions of $Q_{a \ldots c}$ obtained above with the simple inequalities

$$
\tilde{\mathbf{E}} t_{a}^{2} \leq p_{a}, \quad \tilde{\mathbf{E}} t_{a b}^{2} \leq C p_{a} p_{b}, \quad \tilde{\mathbf{E}} t_{a b c}^{2} \leq C p_{a} p_{b} p_{c}, \quad \tilde{\mathbf{E}} t_{a b c d}^{2} \leq C p_{a} p_{b} p_{c} p_{d} .
$$

Here $C$ is an absolute constant. We also use the inequalities $p_{x w} \leq(n m)^{-1 / 2} Y_{x} X_{w}$.

Proof of the bound $Z_{1}=o_{P}\left(S_{Y}^{2}\right)$. We have

$$
Z_{1} \leq \sum_{y \in V} \sum_{w \in W} p_{y w}\left(Q_{(y w) 1}+Q_{(y w) 2}\right)^{2} .
$$

Invoking the inequalities $\left(Q_{(y w) 1}+Q_{(y w) 2}\right)^{2} \leq 2 Q_{(y w) 1}^{2}+2 Q_{(y w) 2}^{2}$ and

$$
\begin{aligned}
Q_{(y w) 1} & \leq \sum_{x \in V \backslash\{y\}} \sum_{\tau \in W \backslash\{w\}} \sum_{z \in V \backslash\{x, y\}} \frac{Y_{x} X_{w}}{\sqrt{n m}} \frac{Y_{x} X_{\tau}}{\sqrt{n m}} \frac{Y_{z} X_{\tau}}{\sqrt{n m}} \leq \frac{X_{w}}{\sqrt{m}} \frac{S_{Y}}{\sqrt{n}} \hat{a}_{2} \hat{b}_{1}, \\
Q_{(y w) 2} & \leq \sum_{x \in V \backslash\{y\}} \sum_{\tau \in W \backslash\{w\}} \sum_{z \in V \backslash\{x, y\}} \frac{Y_{x} X_{w}}{\sqrt{n m}} \frac{Y_{y} X_{\tau}}{\sqrt{n m}} \frac{Y_{z} X_{\tau}}{\sqrt{n m}} \leq \frac{X_{w}}{\sqrt{m}} Y_{y} \sqrt{n} \hat{a}_{2} \hat{b}_{1}^{2},
\end{aligned}
$$

we obtain

$$
Z_{1} \leq 2 \hat{a}_{2}^{2} \hat{b}_{1}^{3} \frac{S_{Y}^{2}}{\sqrt{n}} \frac{S_{X}}{m^{3 / 2}}+2 \hat{a}_{2}^{2} \hat{b}_{1}^{4} \frac{S_{X}}{m^{3 / 2}} \sqrt{n} \sum_{y \in V} Y_{y}^{3} .
$$

Note that $\mathbf{E} X_{1}^{2}<\infty$ implies $S_{X} m^{-3 / 2}=o_{P}(1)$. Furthermore, we have $\hat{a}_{2}, \hat{b}_{1}=O_{P}(1)$. Hence the first summand is $o_{P}\left(S_{Y}^{2}\right)$. To show that the second summand is $o_{P}\left(S_{Y}^{2}\right)$ we use the fact (which follows from $\mathbf{E} Y_{1}^{2}=\infty$ by Lemma 1 ) that $n=o_{P}\left(S_{Y}\right)$ and invoke inequalities

$$
\sum_{y \in V} Y_{y}^{3} \leq S_{Y} \max _{y \in V} Y_{y} \leq S_{Y}\left(S_{Y}\right)^{1 / 2} .
$$

We obtain $\sqrt{n} \sum_{y \in V} Y_{y}^{3}=o_{P}\left(\sqrt{S_{Y}}\right) S_{Y}^{3 / 2}=o_{P}\left(S_{Y}^{2}\right)$. We conclude that $Z_{1}=o_{P}\left(S_{Y}^{2}\right)$.

Proof of the bound $Z_{2}=o_{P}\left(S_{Y}^{2}\right)$. We split $Z_{2}=Z_{21}+Z_{22}+Z_{23}$, where the sum

$$
Z_{21}=\sum_{\{x, y\} \subset V} \sum_{w \in W} \sum_{\tau \in W \backslash\{w\}} t_{(y w)(x \tau)}^{2} Q_{(y w)(x \tau)}^{2}
$$


accounts for pairs of non incident edges $a=(y w)$ and $c=(x \tau)$, while the sums

$$
Z_{22}=\sum_{\{x, y\} \subset V} \sum_{w \in W} t_{(y w)(x w)}^{2} Q_{(y w)(x w)}^{2} \quad \text { and } \quad Z_{23}=\sum_{x \in V} \sum_{\{w, \tau\} \subset W} t_{(x w)(x \tau)}^{2} Q_{(x w)(x \tau)}^{2}
$$

account for pairs of incident edges $a=(y, w), b=(x, w)$ and $b=(x, w), c=(x, \tau)$ respectively. To estimate $Z_{21}$ we use (54) and obtain that

$$
Q_{(y w)(x \tau)} \leq \frac{X_{w} X_{\tau}}{m}\left(Y_{y} \hat{b}_{1}+Y_{x} \hat{b}_{1}+\frac{S_{Y}}{n}\right) .
$$

Hence,

$$
Z_{21} \leq C \frac{S_{X}^{2}}{m^{3}}\left(2 \hat{b}_{1}^{3} \sum_{x \in V} Y_{x}^{3}+\hat{b}_{1}^{2} n^{-1} S_{Y}^{2}\right)
$$

From (56) and the fact that $n=o_{P}\left(S_{Y}\right)$ we conclude that $Z_{21}=o_{P}\left(S_{Y}^{2}\right)$. To estimate $Z_{22}$ and $Z_{23}$ we use the first and second identities of (55). We obtain

$$
Q_{(y w)(x w)} \leq\left(Y_{x}+Y_{y}\right) \hat{a}_{2} \hat{b}_{1} \quad \text { and } \quad Q_{(x w)(x \tau)} \leq 2 \hat{b}_{1}^{2} X_{w} X_{\tau} n m^{-1} .
$$

Hence,

$$
Z_{22} \leq C \hat{a}_{2}^{3} \hat{b}_{1}^{3} \sum_{x \in V} Y_{x}^{3}, \quad Z_{23} \leq C \hat{b}_{1}^{4} S_{X}^{2} m^{-3} S_{Y} n
$$

We note that both quantities on the right are of order $o_{P}\left(S_{Y}^{2}\right)$, since $n=o_{P}\left(S_{Y}\right)$ by Lemma 1 and $S_{X}^{2}=o_{P}\left(m^{3}\right)$. We conclude that $Z_{2}=o_{P}\left(S_{Y}^{2}\right)$.

Proof of the bound $Z_{3}=o_{P}\left(S_{Y}^{2}\right)$. We split $Z_{3}=Z_{31}+Z_{32}$, where

$$
\begin{aligned}
& Z_{31}=\sum_{y \in V} \sum_{w \in W} \sum_{x \in V \backslash\{y\}} \sum_{\tau \in W \backslash\{w\}} t_{(y w)(x w)(x \tau)}^{2}\left(\sum_{z \in V \backslash\{x, y\}} p_{z \tau}\right)^{2}, \\
& Z_{32}=\sum_{\{x, y\} \subset V} \sum_{w \in W} \sum_{\tau \in W \backslash\{w\}} \sum_{z \in V \backslash\{x, y\}} t_{(y w)(x w)(z \tau)}^{2}\left(p_{y \tau}+p_{x \tau}\right)^{2} .
\end{aligned}
$$

We have

$$
Z_{31} \leq C \hat{a}_{2} \hat{b}_{1}^{3} \frac{S_{X}}{m^{3 / 2}} \sqrt{n} S_{Y}, \quad Z_{32} \leq C \hat{a}_{2} \hat{b}_{1}^{2} \frac{S_{X}}{m^{3 / 2}} n^{-1 / 2} \sum_{x \in V} Y_{x}^{3}
$$

By the same argument as above we obtain that $Z_{3}=o_{P}\left(S_{Y}^{2}\right)$.

Finally, we have

$$
Z_{4} \leq \sum_{x \in V} \sum_{y \in V \backslash\{x\}} \sum_{z \in V \backslash\{x, y\}} \sum_{w \in W} \sum_{\tau \in W \backslash\{w\}} t_{(y w)(x w)(x \tau)(z \tau)}^{2} \leq C \hat{a}_{2}^{2} \hat{b}_{1}^{2} S_{Y}=O_{P}\left(S_{Y}\right)=o_{P}\left(S_{Y}^{2}\right) .
$$

The proof of the bound $\tilde{\mathbf{E}}\left(N_{2}-\tilde{\mathbf{E}} N_{2}\right)^{2}=o_{P}\left(S_{Y}^{2}\right)$ is completed.

Proof of (ii). Denoting the sum $\sum_{\Lambda} \sum_{w \in W} \sum_{\tau \in W \backslash\{w\}}$ by $\sum_{*}$ and using the shorthand notation

$$
\begin{aligned}
& \mathbb{I}^{*}=\mathbb{I}_{x w} \mathbb{I}_{y w} \mathbb{I}_{x \tau} \mathbb{I}_{z \tau}, \quad p^{*}=p_{x w} p_{y w} p_{x \tau} p_{z \tau}, \quad \lambda^{*}=\lambda_{x w} \lambda_{y w} \lambda_{x \tau} \lambda_{z \tau}, \\
& \delta_{1}^{*}=\left(\lambda_{x w}-p_{x w}\right) \lambda_{y w} \lambda_{x \tau} \lambda_{z \tau}, \quad \delta_{2}^{*}=p_{x w}\left(\lambda_{y w}-p_{y w}\right) \lambda_{x \tau} \lambda_{z \tau}, \\
& \delta_{3}^{*}=p_{x w} p_{y w}\left(\lambda_{x \tau}-p_{x \tau}\right) \lambda_{z \tau}, \quad \delta_{4}^{*}=p_{x w} p_{y w} p_{x \tau}\left(\lambda_{z \tau}-p_{z \tau}\right),
\end{aligned}
$$

we have $N_{2}=\sum_{*} \mathbb{I}^{*}$ and $\tilde{\mathbf{E}} \mathbb{I}^{*}=p^{*}$, and $\tilde{\mathbf{E}} N_{2}=\sum_{*} p^{*}$. 
We derive (ii) from the relations shown below

$$
\sum_{*} p^{*}=\left(1+o_{P}(1)\right) \sum_{*} \lambda^{*} \quad \text { and } \quad \sum_{*} \lambda^{*}=2^{-1} \hat{a}_{2}^{2} \hat{b}_{1}^{2} S_{Y}+o_{P}\left(S_{Y}\right) .
$$

To prove the second relation we regroup the sum

$$
\begin{aligned}
\sum_{*} \lambda^{*} & =\sum_{\Lambda} Y_{x}^{2} \frac{Y_{y} Y_{z}}{n^{2}}\left(\hat{a}_{2}^{2}-\sum_{w \in W} \frac{X_{w}^{4}}{m^{2}}\right) \\
& =\frac{1}{2} \sum_{x \in V} Y_{x}^{2}\left(\left(\hat{b}_{1}-\frac{Y_{x}}{n}\right)^{2}-\sum_{z \in V \backslash\{x\}} \frac{Y_{z}^{2}}{n^{2}}\right)\left(\hat{a}_{2}^{2}-\sum_{w \in W} \frac{X_{w}^{4}}{m^{2}}\right) .
\end{aligned}
$$

For $\mathbf{E} X_{1}^{2}<\infty$ and $\mathbf{E} Y_{1}<\infty$, we obtain from Lemma 3 that

$$
\sum_{w \in W} \frac{X_{w}^{4}}{m^{2}}=o_{P}(1), \quad \sum_{z \in V} \frac{Y_{z}^{2}}{n^{2}}=o_{P}(1), \quad \sum_{x \in V} \frac{Y_{x}^{3}}{n}=o_{P}\left(S_{Y}\right), \quad \sum_{x \in V} \frac{Y_{x}^{4}}{n^{2}}=o_{P}\left(S_{Y}\right) .
$$

Invoking these bounds in (58) we obtain the second relation of (57).

To prove the first bound of (57) we write

$$
\begin{aligned}
& 0 \leq \sum_{*} \lambda^{*}-\sum_{*} p_{*}=\sum_{*}\left(\delta_{1}^{*}+\delta_{2}^{*}+\delta_{3}^{*}+\delta_{4}^{*}\right) \leq \sum_{*} \lambda^{*}\left(\mathbb{I}_{1}^{*}+\mathbb{I}_{2}^{*}+\mathbb{I}_{3}^{*}+\mathbb{I}_{4}^{*}\right), \\
& \mathbb{I}_{1}^{*}:=\mathbb{I}_{Y_{x} X_{w}>\sqrt{n m}}, \quad \mathbb{I}_{2}^{*}:=\mathbb{I}_{Y_{y} X_{w}>\sqrt{n m}}, \quad \mathbb{I}_{3}^{*}:=\mathbb{I}_{Y_{x} X_{\tau}>\sqrt{n m}}, \quad \mathbb{I}_{4}^{*}:=\mathbb{I}_{Y_{z} X_{\tau}>\sqrt{n m}}
\end{aligned}
$$

and estimate $\sum_{*} \lambda^{*} \mathbb{I}_{i}^{*}=o_{P}\left(S_{Y}\right)$, for $i \in[4]$. We only show this bound for $i=1$. Let $\varepsilon(\cdot)$ be the function associated with the distribution of $Y_{1}$ by Lemma 3. So that $\varepsilon(n)=o(1)$ and with probability $1-o_{P}(1)$ we have $\max _{x \in V} Y_{x} \leq n \varepsilon(n)$. If the latter inequality holds, then every event $\mathbb{I}_{Y_{x} X_{w}>\sqrt{n m}}=1$ implies $X_{w}>\beta_{n}^{1 / 2} \varepsilon^{-1}(n)$. We denote the indicator of the latter event $\mathbb{I}_{w}$. We have with probability $1-o(1)$

$$
\sum_{*} \lambda^{*} \mathbb{I}_{1}^{*} \leq \sum_{\Lambda} Y_{x}^{2} \frac{Y_{y} Y_{z}}{n^{2}} \sum_{w \in W} \frac{X_{w}^{2}}{m} \mathbb{I}_{w} \sum_{\tau \in W \backslash\{w\}} \frac{X_{\tau}^{2}}{m} \leq 2^{-1} \hat{a}_{2} \hat{b}_{1}^{2} S_{Y} \sum_{w \in W} \frac{X_{w}^{2}}{m} \mathbb{I}_{w} .
$$

Finally, $\mathbf{E} X_{1}^{2}<\infty$ implies $\mathbf{E} \sum_{w \in W} \frac{X_{w}^{2}}{m} \mathbb{I}_{w}=o(1)$. Hence $\sum_{w \in W} \frac{X_{w}^{2}}{m} \mathbb{I}_{w}=o_{P}(1)$.

\section{References}

[1] Bloznelis, M.: Degree and clustering coefficient in sparse random intersection graphs, The Annals of Applied Probability 23 (2013), 1254-1289.

[2] Bloznelis, M., Damarackas, J.: Degree distribution of an inhomogeneous random intersection graph. Electron. J. Comb. 20(3) (2013), \#P3.

[3] Bloznelis, M., Godehardt, E., Jaworski, J., Kurauskas, V., Rybarczyk, K.: Recent Progress in Complex Network Analysis - Models of Random Intersection Graphs. In: Lausen, B., Krolak-Schwerdt, S., Böhmer, M. (eds) Data Science, Learning by Latent Structures, and Knowledge Discovery, Springer, Berlin, (2015), 69-78. 
[4] Bloznelis, M., Godehardt, E., Jaworski, J., Kurauskas, V., Rybarczyk, K.: Recent Progress in Complex Network Analysis - Properties of Random Intersection Graphs. In: Lausen, B., Krolak-Schwerdt, S., Böhmer, M. (eds) Data Science, Learning by Latent Structures, and Knowledge Discovery, Springer, Berlin, (2015), 79-88.

[5] Bloznelis, M., Kurauskas, K.: Clustering function: another view on clustering coefficient. Journal of Complex Networks 4 (2016), 61-86.

[6] Deijfen, M., Kets, W.: Random intersection graphs with tunable degree distribution and clustering, Probab. Engrg. Inform. Sci. 23 (2009), 661-674.

[7] Feller, W.: An introduction to probability theory and its applications. Vol. II. 2nd Edition. John Wiley \& Sons, New York, (1971).

[8] Foss, S., Korshunov, D., Zachary, S.: An Introduction to Heavy-Tailed and Subexponential Distributions. 2nd Edition. Springer, New York, (2013).

[9] Godehardt, E., Jaworski, J.: Two models of random intersection graphs for classification, in: Studies in Classification, Data Analysis and Knowledge Organization, Springer, Berlin, (2003), 67-81.

[10] Godehardt, E., Jaworski, J., Rybarczyk, K.: Clustering coefficients of random intersection graphs, in: Studies in Classification, Data Analysis and Knowledge Organization, Springer, Berlin, (2012), 243-253.

[11] Jacob, E., Mörters, P.: A spatial preferential attachment model with local clustering, In: Bonato, A., Mitzenmacher, M., Prałat, P. (eds) Algorithms and Models for the Web Graphs, WAW2013, LNCS 8305, Springer, Berlin, (2013), 14-25.

[12] Karoński, M., Scheinerman, E. R., Singer-Cohen, K. B.: On random intersection graphs: The subgraph problem, Combinatorics, Probability and Computing 8 (1999), 131-159.

[13] Kurauskas, V.: On local weak limit and subgraph counts for sparse random graphs, arXiv:1504.08103v2, (2015).

[14] Newman, M. E. J., Strogatz, S. H., Watts, D. J.: Random graphs with arbitrary degree distributions and their applications, Physical Review E 64 (2001) 026118.

[15] Newman, M. E. J.: The structure of scientific collaboration networks, Proc. Natl.Acad. Sci. USA 98 (2001), 404-409.

[16] Newman, M. E. J.: Scientific collaboration networks. I and II, Physical Review E 64 (2001) 016131, 016132.

[17] Ostroumova Prokhorenkova, L., Samosvat, E.: Global clustering coefficient in scale-free networks, In: Bonato, A., Chung Graham, F., Prałat, P. (eds) Algorithms and Models for the Web Graphs, WAW2014, LNCS 8882, Springer, Berlin, (2014), 47-58.

[18] Redner, S.: How popular is your paper? An empirical study of the citation distribution, Eur.Phys. J. B 4. (1998), 131-134. 